\title{
E2F1 mediates sustained lipogenesis and contributes to hepatic steatosis
}

\author{
Pierre-Damien Denechaud, ${ }^{1}$ Isabel C. Lopez-Mejia, ${ }^{1}$ Albert Giralt, ${ }^{1}$ Qiuwen Lai, ${ }^{1}$ Emilie Blanchet, ${ }^{2}$ Brigitte Delacuisine, ${ }^{1}$ \\ Brandon N. Nicolay, ${ }^{3}$ Nicholas J. Dyson, ${ }^{3}$ Caroline Bonner, ${ }^{4,5,6}$ François Pattou, ${ }^{4,5,6}$ Jean-Sébastien Annicotte, ${ }^{2,7}$ and Lluis Fajas ${ }^{1}$ \\ 'Department of Physiology, Université de Lausanne (UNIL), Lausanne, Switzerland. Institut de Cénétique Moléculaire de Montpellier, Université de Montpellier, Montpellier, France. ${ }^{3}$ Laboratory of Molecular \\ Oncology, Massachusetts Ceneral Hospital Cancer Center, Harvard Medical School, Charlestown, Massachusetts, USA. "European Genomic Institute for Diabetes, Lille, France. ${ }^{5}$ INSERM UMR 1190, Lille, \\ France. ${ }^{6}$ Centre Hospitalier Régional Universitaire, Lille, France. 'European Cenomic Institute for Diabetes, Université Lille Nord de France, UMR 8199 CNRS, Lille, France.
}

\begin{abstract}
E2F transcription factors are known regulators of the cell cycle, proliferation, apoptosis, and differentiation. Here, we reveal that E2F1 plays an essential role in liver physiopathology through the regulation of glycolysis and lipogenesis. We demonstrate that E2F1 deficiency leads to a decrease in glycolysis and de novo synthesis of fatty acids in hepatocytes. We further demonstrate that E2F1 directly binds to the promoters of key lipogenic genes, including Fasn, but does not bind directly to genes encoding glycolysis pathway components, suggesting an indirect effect. In murine models, E2F1 expression and activity increased in response to feeding and upon insulin stimulation through canonical activation of the CDK4/pRB pathway. Moreover, E2F1 expression was increased in liver biopsies from obese, glucose-intolerant humans compared with biopsies from lean subjects. Finally, E2f1 deletion completely abrogated hepatic steatosis in different murine models of nonalcoholic fatty liver disease (NAFLD). In conclusion, our data demonstrate that E2F1 regulates lipid synthesis and glycolysis and thus contributes to the development of liver pathology.
\end{abstract}

\section{Introduction}

E2F transcription factors are the ultimate effectors of distinct signaling cascades that regulate the expression of genes involved in cellular homeostasis. E2Fs exist either as heterodimers associated with dimerization partner (DP) proteins or with larger complexes including members of the retinoblastoma family of proteins (pRBs) that includes RB1, RBL1, and RBL2 (1). In general, the association of E2Fs with $\mathrm{pRB}$ family members induces the repression of target genes. When phosphorylated by active cyclin-cyclin-dependent kinase (CDK) complexes, pRBs are released, enabling E2Fs to drive transcriptional regulation. In proliferating cells, E2F target genes include effectors of DNA replication, mitosis, DNA repair, and apoptosis (1).

Of particular interest is the observation that E2F1, the first-described and most-studied member of the E2F family, has important metabolic functions beyond the control of the cell cycle in nonproliferating cells. Indeed, we recently demonstrated, both in vitro and in vivo, that E2F1 directly regulates the expression of Kir6.2, a key component of the KATP channel that is involved in the regulation of glucose-induced insulin secretion in nonproliferating pancreatic $\beta$ cells (2). E2F1 was also implicated in the regulation of adipose tissue metabolism through transcriptional regulation of the master adipogenic factor PPAR $\gamma$ during early stages of adipogenesis (3). E2F1 is also involved in metabolic functions in other tissues, such as muscle and brown adipose tissue, in which E2F1 modulates oxidative metabolism (4). Likewise, other studies have implicated E2F1 in the control of glucose homeostasis. For example, E2F1

Conflict of interest: The authors have declared that no conflict of interest exists. Submitted: February 17, 2015; Accepted: October 22, 2015.

Reference information: / Clin Invest. 2016;126(1):137-150. doi:10.1172/JCI81542. directly regulates the gene encoding pyruvate dehydrogenase kinase 4 (PDK4), a key nutrient sensor and modulator of glucose oxidation, with the net result of restricting mitochondrial glucose oxidation (5). Moreover, 6-phosphofructo-2-kinase/ fructose-2,6-bisphosphatase, the glycolytic enzyme involved in cell proliferation, was identified as an E2F target gene (6).

Overall, the above studies suggest that E2F1 contributes to whole-body metabolic homeostasis via distinct roles in different metabolic tissues. Studies concerning the retinoblastoma protein RB1 further support a major role of E2F1 in metabolism (7-9).

Although the liver plays a central role in whole-body glucose and lipid homeostasis, studies of the role of E2F1 in this tissue are limited to its participation in proliferation and oncogenesis (10, 11). Therefore, the function of E2F1 in liver metabolism remains to be explored. During fasting, the liver sustains a constant energy substrate supply for the organism by producing glucose from glycogenolysis and gluconeogenesis. In contrast, during the fed state, glucose enters the liver and is rapidly metabolized to replenish glycogen stores. Excess glucose is then converted into triglycerides (TG) via de novo lipogenic pathways (12).

In some metabolic disorders, including obesity, the liver exhibits increased glycolysis and lipid synthesis, resulting in the typical accumulation of fat in the liver (hepatic steatosis) (12). Genes involved in liver glycolysis and lipogenesis are regulated at the transcriptional level in response to insulin and glucose through the transcription factor sterol regulatory element-binding protein 1c (SREBP1c) and carbohydrate-responsive element-binding protein (CHREBP), respectively. Insulin, by activating SREBP1c, induces glucokinase (GCK) expression, which is the rate-limiting enzyme of glycolysis in the liver, thus controlling the glucose flux $(13,14)$. CHREBP, similar to SREBP1c, triggers the expression of genes such as acetyl-CoA 
A
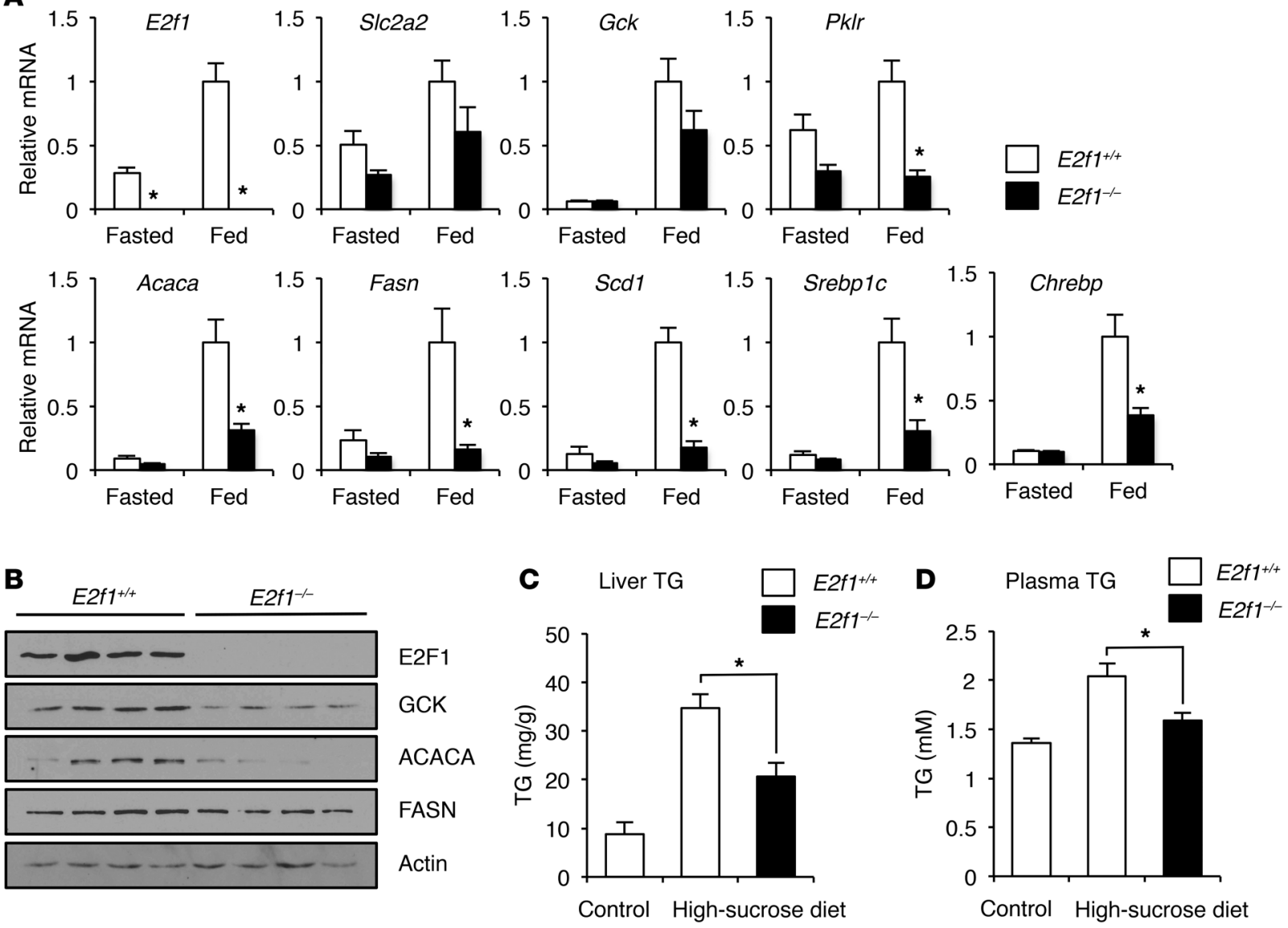

Figure 1. E2f1-/- mice show a decrease in liver glucose and lipid metabolism. (A) Relative mRNA levels of E2f1 and relevant glycolytic and lipogenic genes in the livers of $E 2 \mathrm{fl}^{+/+}$versus $E 2 \mathrm{f1}^{-/-}$mice ( $n=5$ per group) under fed conditions. HFruct, high-fructose. (B) Western blot analyses of the expression of the

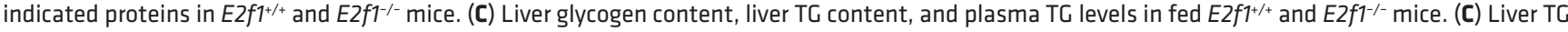
content in $E 2 \mathrm{f1}^{+/+}$and $E 2 f 1^{-/-}$mice subjected to a 9-week high-sucrose diet (6 mice per group). (D) Plasma TG levels after overnight fasting of $E 2 f 1^{+/+}$and E2f1-/- mice subjected to a 9-week high-sucrose diet (6 mice per group). ${ }^{*} P<0.05$, by 2 -tailed, unpaired $t$ test.

carboxylase (ACACA), fatty acid synthase (FASN), and stearoyl CoA desaturase (SCD1), which drive lipogenesis in the liver (15).

In this study, we demonstrate that E2F1 is essential for metabolic homeostasis in the liver through the control of glycogenic and lipogenic pathways. In particular, we show that E2F1 deficiency protects against obesity- and diabetes-induced liver steatosis in mouse models. Furthermore, we show that E2F1 expression is substantially increased in the liver of obese human subjects.

\section{Results}

E2F1 deficiency results in impaired glucose and lipid metabolism in the liver. To investigate the role of E2F1 in liver metabolism, we initially analyzed changes in glucose and lipid homeostasis in the livers of $E 2 \mathrm{fl}^{-/-}$mice. No major differences were observed in glucose or insulin levels in the plasma of $E 2 \mathrm{fl}^{-/-}$compared with that of $E 2 \mathrm{fl}^{+/+}$mice in the fed state (Supplemental Figure 1, A and B; supplemental material available online with this article; doi:10.1172/ JCI81542DS1). Gene expression analyses indicated, however, that liver glycolytic pyruvate kinase (Pklr) and lipogenic Acaca, Fasn, and $S c d 1$ mRNA levels were decreased in $E 2 \mathrm{fl}^{-/-}$compared with levels in $E 2 \mathrm{Fl}^{+/+}$mice (Figure 1A). Moreover, decreased mRNA levels correlated with reduced ACACA, FASN, and GCK protein expression in the livers of these mice (Figure 1B). The glucose/ lipid pathway is transcriptionally regulated by multiple transcription factors, including SREPB1c and CHREBP, which function as insulin and glucose sensors, respectively. Interestingly, Srebp1c and Chrebp mRNA expression levels were strongly decreased in $\mathrm{E} 2 \mathrm{fl}^{-/-}$mice compared with levels in $\mathrm{E} \mathrm{fl}^{+/+}$mice (Figure $\left.1 \mathrm{~A}\right)$. Surprisingly, no major differences were observed in E2F1-deficient mice fed a normal chow diet in terms of GCK activity, glycogen phosphorylase activity, or glycogen and TG content in the liver (Supplemental Figure 1, C-G). In the fasted state, we did not observe a significant reduction in the expression of glycolytic or lipogenic genes (Figure 1A).

Lipid homeostasis was also compromised by E2F1 deficiency. $E 2 \mathrm{fl}^{+/+}$and $E 2 \mathrm{fl}^{-/-}$mice were subjected to a high-sucrose dietary regime. The typical accumulation of TG observed in the liver in these conditions was reduced by $40 \%$ in $E 2 \mathrm{f1}^{-/-}$mice compared with the content found in their WT littermates (Figure $1 C$ ). We also observed a modest yet significant decrease in circulating TG levels in $E 2 \mathrm{fl}^{-/-}$mice in response to a high-sucrose diet (Figure 1D). These results were independent of any significant difference in body weight (BW), glycemia, insulinemia, or plasma free fatty acid (FFA) levels (Supplemental Figure 1, 
$\mathrm{H}-\mathrm{K}$ ), suggesting that the observed effects in the absence of E2F1 were liver cell autonomous.

To demonstrate that the effects of E2f1 deletion observed in the liver were effectively tissue autonomous, we generated E2f1-floxed mice on a C57BL/6 background. E2f1-floxed mice were crossed with Alb-Cre mice to delete E2f1 specifically in the liver (Alb-Cre E2f1 $1^{f / f l}$, herein referred to as E2F1 LKO mice). We observed no differences in glycolytic or lipogenic gene expression in mice on a chow diet (Figure 2A); therefore, we decided to challenge these mice with a high-fructose diet to stimulate lipogenesis (16). Like E2f1/- mice, E2F1 LKO mice showed a significant reduction in expression levels of glucose transporter 2 (Slc2a2), Gck, Pklr, Acaca, Fasn, Scd1, and Srebp1c genes in liver (Figure 2A). Subsequently, liver weight, liver TG content, and plasma TG levels were reduced in E2F1 LKO mice compared with $E 2 f 1^{f / f l}$ mice (Figure 2B).

E2F1 regulates glycolysis and lipogenesis in hepatocytes. To identify the molecular mechanisms involved in glucose and lipid homeostasis regulation by E2F1, we decided to use the model of primary cultures from mouse hepatocytes. As expected, the expression of Slc2a2, Gck, Pklr, Acaca, Fasn, Scd1, Srebp1c, and Chrebp mRNAs was increased in response to glucose and insulin in $E 2 \mathrm{f1}^{+/+}$hepatocytes (Figure 3A). This effect mimicked the observed changes in the liver under fed conditions (Figure 1A). In contrast, mRNA expression of these genes was not properly induced in response to glucose and insulin in $E 2 \mathrm{f1}^{-/-}$hepatocytes (Figure 3A). In addition, similar to the previous observation in liver (Figure 1B), GCK, ACACA, and FASN protein levels were also decreased in $E 2 \mathrm{fl}^{-/-}$compared with those in $E 2 \mathrm{fi}^{+/+}$cells (Figure 3B). These results suggested that E2F1 regulates the glycolytic and lipogenic gene expression programs in hepatocytes. Furthermore, in comparison with $E 2 \mathrm{f1}^{+/+}$cells, we found that decreased mRNA expression of key glycolytic genes correlated with decreased glycolysis in primary $E 2 f^{-1 /}$ hepatocytes, as measured by the extracellular acidification rate (ECAR) upon glucose injection into a Seahorse analyzer (Figure 4A). Consequently, there was a decrease in lactate concentration in the culture medium (Supplemental Figure 2A). Because E2F1 activity has previously been implicated in increased glucose oxidation in the heart (5), we verified that the reduced glycolysis observed in $E 2 f^{-{ }^{--}}$hepatocytes was not the result of increased glucose oxidation and/or PDH activity. No differences in PDH activity were observed (Supplemental Figure 2, B and C), suggesting that impaired glycolysis, due in part to diminished GCK expression (and activity), contributed to a robust reduction of glycogen content in $E 2 \mathrm{fl}^{-/-}$hepatocytes after glucose-insulin stimulation (Figure 4B). This decrease in glycogen content could also be correlated with reduced expression levels of glycogen synthase and glycogen phosphorylase in E2f1 ${ }^{-/}$hepatocytes (Supplemental Figure 2, D and E). De novo lipid synthesis, which is typically observed when glucose levels are high, was also decreased in both basal and glucose-insulinstimulated $E 2 \mathrm{fl}^{-{ }^{--}}$hepatocytes (Figure $4 \mathrm{C}$ ). Hence, we observed less lipid accumulation, as measured by Oil Red O staining, in E2f1- compared with E2f1 ${ }^{+/+}$hepatocytes (Figure 4D). Taken together, these results suggested that the impaired glycolysis and lipid synthesis that we observed in the livers of $E 2 \mathrm{fl}^{-/-}$mice were due to the specific function of $\mathrm{E} 2 \mathrm{~F} 1$ in hepatocytes.

\section{Table 1. E2F1 ChIP-seq data analysis}

\section{Enrichment of genes bound by E2F1}

\begin{tabular}{lc} 
Biological processes & $\boldsymbol{P}$ value \\
Cellular metabolic process & $2.5 \times 10^{-87}$ \\
\hline Primary metabolic process & $3.5 \times 10^{-68}$ \\
\hline Macromolecule metabolic process & $1.3 \times 10^{-65}$ \\
\hline Nitrogen compound metabolic process & $1.0 \times 10^{-54}$ \\
Organelle organization & $2.6 \times 10^{-37}$ \\
Cell cycle & $1.3 \times 10^{-36}$ \\
Biosynthetic process & $9.9 \times 10^{-30}$ \\
Cell-cycle process & $9.8 \times 10^{-25}$ \\
Cell division & $2.9 \times 10^{-23}$ \\
Cellular response to stimulus & $1.8 \times 10^{-22}$ \\
Regulation of metabolic process & $3.1 \times 10^{-16}$
\end{tabular}

Enrichment of genes associated with E2F1 DNA binding using Gene Ontology Biological Processes (GOTERM_BP2) analysis.

E2F1 regulates global lipogenic and glycolytic gene expression programs in the liver. Our results indicated that E2F1 plays an important role in supporting glucose and lipid metabolism in the liver. The major function of E2F1 is to regulate gene transcription. Thus, to elucidate the mechanism underlying the effects of E2F1 depletion in the liver, we performed ChIP high-throughput DNA sequencing (ChIP-seq) in a primary culture of mouse hepatocytes infected with adenovirus-E2F1 (Ad-E2F1) using a validated anti-E2F1 Ab (Supplemental Figure 3, A and B) to identify E2F1 target genes. Experimental replicates were validated as described in Methods (Supplemental Figure 3, C and D). Sequencing alignment to the Ensembl Mouse Assembly NCBIM37 (mm9), peak detection, and peak assignment to genes was performed using the High-throughput Sequencing Data Analysis portal of the HTSstation (17). As expected, E2F1 target cell-cycle genes, including Ccne1, Ccna2, Rb1, Rbl1, Cdkn2a, $T k 1$, and Dhfr were found (Supplemental Figure 4). Consistent with previous studies (18), we found that E2F1 preferentially binds to promoter regions and that the major E2F1 motif enrichment is GCGCGC (Supplemental Figure 5, A-C). We used the Database for Annotation, Visualization, and Integrated Discovery (DAVID) to build a cluster of genes bound by E2F1 with the Gene Ontology Biological Processes (GOTERM_BP2) (http://www.geneontology.org) database. As expected, the cluster of genes bound by E2F1 that were involved in the cell cycle, cell division, and cell-cycle processes was well represented (Table 1 ).

Most interesting was the finding that the most significant cluster of genes was related to cellular metabolic processes (Table 1). This supported our hypothesis that E2F1 may be an important regulator not only of the cell cycle but also of cellular metabolism. Lipogenesis and glycolysis are major metabolic processes that take place in the liver, and our statistical analysis focusing on liverspecific genes also showed that the cluster of genes involved in fatty acid metabolism were significantly represented in the GOTERM_BP2 and KEGG_Pathway (Kyoto Encyclopedia of Genes and Genomes) (http://www.genome.jp/kegg/pathway. html) databases (Supplemental Figure 6). In a more precise analysis of the E2F1 ChIP-seq data, E2F1 was found to be bound to the 

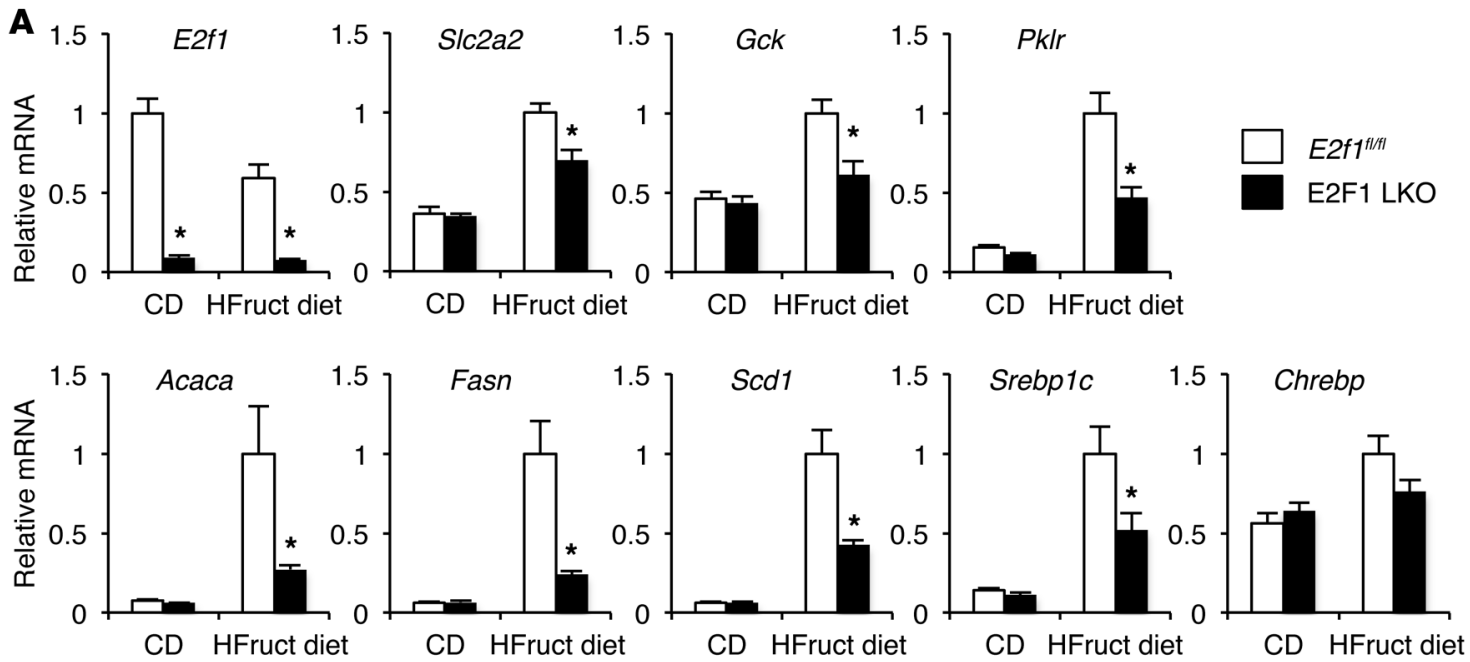

B

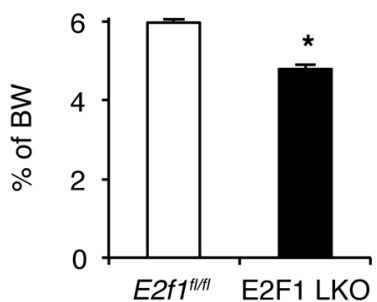

Liver TG

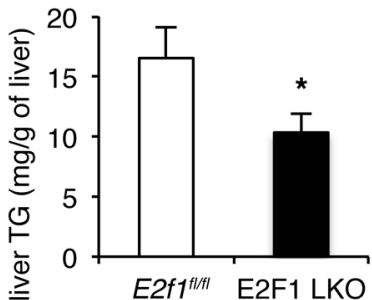

Plasma TG

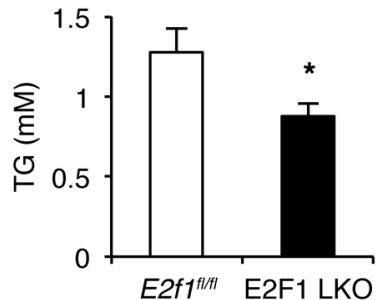

Figure 2. Liver-specific deletion of E2f1 leads to decreased glycolytic and lipogenic programs. (A) Relative mRNA levels of E2f1 and relevant glycolytic and lipogenic genes in the livers E2f1/ffflversus E2F1 LKO mice on a chow diet or after a 2-week high-fructose diet ( $n=5$ per group). (B) Liver weight, liver TC content, and plasma TC levels of E2f1/f/fl versus E2F1 LKO mice after 2 weeks on a high-fructose diet $\left(n=5\right.$ per group). ${ }^{*} P<0.05$, by 2-tailed, unpaired $t$ test.

promoters of Fasn, Chrebp, and Srebp1c (Supplemental Figure 4). In addition, ChIP-quantitative PCR (ChIP-qPCR) experiments showed that E2F1 directly binds the promoters of the lipogenic Acaca and $S c d 1$ genes and confirmed that E2F1 binds the promoters of Fasn and of the transcription factors Srebp1c and Chrebp (Figure $5 \mathrm{~A}$ ). In contrast, we did not detect any significant chromatin enrichment in the promoters of the glycolytic genes $S l c 2 a 2, G c k$, or Pklr (Figure 5A). The same pattern of DNA binding was observed in HepG2 human hepatoma cells lines when we performed ChIP on endogenous E2F1 (Supplemental Figure 7A). E2F1 overexpression in hepatocytes was able to induce Acaca, Fasn, and Srebp1c gene expression (Supplemental Figure 7B). In contrast, other genes we identified in the E2F1 ChIP analysis were not induced by E2F1 overexpression, suggesting that other E2F1 partners are required. The functional occupancy of E2F1 in these genes was assessed using luciferase-based reporter studies. E2F1 induced luciferase activity for the Fasn, Scd1, Srebp1c, and Chrebp promoters, but not for the Slc2a2 or Gck promoters (Figure 5B). This suggested that E2F1 regulates the transcriptional activity of key genes including, but not limited to, Fasn, Scd1, Chrebp, and Srebp1c. In addition, through the regulation of Srebp1c and Chrebp transcription, E2F1 also indirectly regulated glycolysis (SLC2A2, GCK, PKLR) and lipogenesis (ACACA, FASN, SCD1) (Figure 5C).

To further characterize the action of E2F1 on the transcription of lipogenic genes, we decided to focus on the Fasn gene, which encodes for the enzyme that catalyzes the rate-limiting reaction in fatty acid synthesis. Sequential deletions of the murine Fasn promoter determined the location of the E2F responsive element (E2FRE). Deletion of this responsive element abrogated the response to E2F1 (Supplemental Figure 8, A-C). It is still possible, however, that removal of the E2FRE in the Fasn promoter also abrogates the SREBP-mediated regulation of the promoter, since the E2FRE is located close to the sterol regulatory element (SRE) in this gene.

The transcription factor upstream stimulatory factor 1 (USF1) typically regulates the transcription of lipogenic genes in response to feeding and insulin signaling and cooperates with SREBP1c to induce Fasn gene transcription (19). We did not observe a synergistic effect of E2F1 on SREBP1 transactivation of the murine Fasn promoter luciferase reporter, suggesting that SREBP1 and E2F1 independently regulate this promoter (Supplemental Figure 9A). Interestingly, USF1 synergized with E2F1 to regulate the activity of the E2F1 synthetic target gene promoter (Figure 5D) and also of the Fasn promoter (Figure 5E). Co-IP experiments showed that USF1 and its heteropartner USF2 were associated with E2F1 (Figure 5F and Supplemental Figure 9B). These results suggest that, similar to other lipogenic transcription factors, E2F1 activity is also regulated by interactions with USF1. These results also demonstrate that E2F1 is a true and direct regulator of the transcription of key genes that participate in lipogenic pathways in the liver (Figure 5C). 


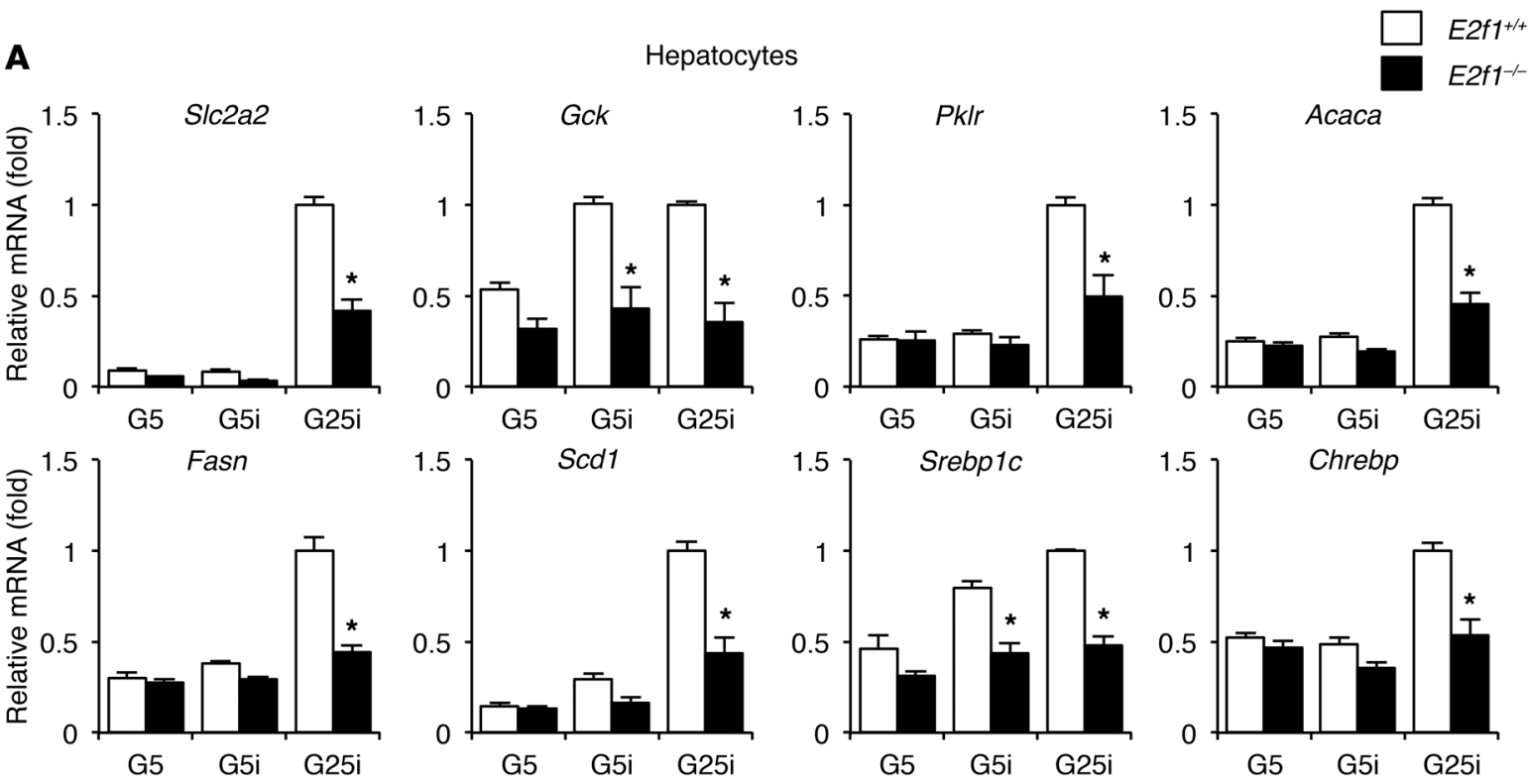

B

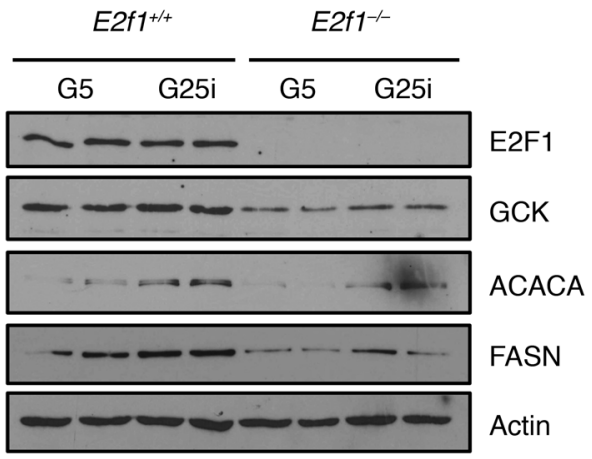

Western blot quantification

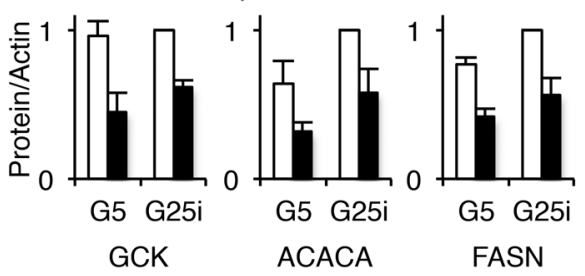

Figure 3. Expression of glycolytic and lipogenic genes is impaired in E2f1 ${ }^{-1-}$ hepatocytes. (A) Relative mRNA expression of relevant glycolytic and lipogenic genes in primary hepatocytes from $E 2 \mathrm{f1}^{+/+}$and $E 2 \mathrm{f1}^{-/-}$mice treated for 24 hours with $5 \mathrm{mM}$ low glucose alone (G5); $5 \mathrm{mM}$ low glucose and $100 \mathrm{nM}$ insulin (G5i); or $25 \mathrm{mM}$ high glucose and $100 \mathrm{nM}$ insulin (G25i). (B) Western blot analysis shows expression levels of the indicated proteins in $E 2 \mathrm{f}^{+/+}$and $E 2 \mathrm{f1}^{-/-}$hepatocytes treated for 24 hours with $\mathrm{G} 5$ or $\mathrm{C} 25 \mathrm{i}$. All experiments represent the average of 3 independent experiments. ${ }^{*} P<0.05$ compared with control, by 2-tailed, unpaired $t$ test.

The CDK4/RB/E2F1 pathway is regulated by insulin in hepatocytes. The expression of lipogenic and glycolytic genes in the liver is glucose and insulin sensitive. Interestingly, we found that E2f1 mRNA and protein expression levels in mouse livers were increased in response to refeeding conditions, suggesting that $\mathrm{E} 2 \mathrm{~F} 1$ regulates lipogenic gene expression in response to glucose and insulin in this tissue (Figure 6, A and B). Indeed, our data showed that insulin induced mRNA and protein expression of E2F1 in primary mouse hepatocytes (Supplemental Figure 10, A and B). Moreover, E2F1 transcriptional activity was also increased in response to insulin, as measured by promoter-reporter luciferase-based assays (Figure 6C). E2F1 transcriptional activity is modulated by nonphosphorylated RB1, which binds to and represses E2F1 activity. Upon phosphorylation, RB1 is released from E2F1 complexes, thereby eliciting the transcription of E2F1 target genes. Similar to canonical E2F1 target genes, Fasn transcription was regulated in an RB1dependent manner: E2F1 transcriptional activation of the Fasn promoter was blunted by RB1, suggesting that FASN is a bona fide
E2F1 target (Figure 6D). Moreover, RB1 had no effect on the basal activity of the Fasn promoter in the absence of E2F1 (Figure 6D and Supplemental Figure 10C). Furthermore, RB1 protein was phosphorylated in response to refeeding or insulin stimulation in liver and hepatocytes, respectively (Figure 6, E and F), and this result was consistent with increased E2F1 activity in response to insulin in these cells (Figure 6C). Furthermore, IP experiments in HepG2 hepatocytes showed that RB1 dissociated from E2F1 upon insulin stimulation in hepatocytes (Figure 6G).

$\mathrm{RB} 1$ dissociates from $\mathrm{E} 2 \mathrm{~F} 1$ when it is phosphorylated by members of the CDK family. We have previously shown that CDK4 kinase activity is induced by insulin in pancreatic $\beta$ cells (2), suggesting that insulin could also regulate CDK4 activity in hepatocytes, leading to induction of E2F1 activity in these cells. Indeed, inhibition of CDK4 by shRNA in hepatocytes blocked RB1 Ser780 phosphorylation (Supplemental Figure 10D) and resulted in the attenuation of glucose and insulin effects on ACACA and FASN mRNA and protein expression levels (Figure 6, $\mathrm{H}$ and I). These 
A
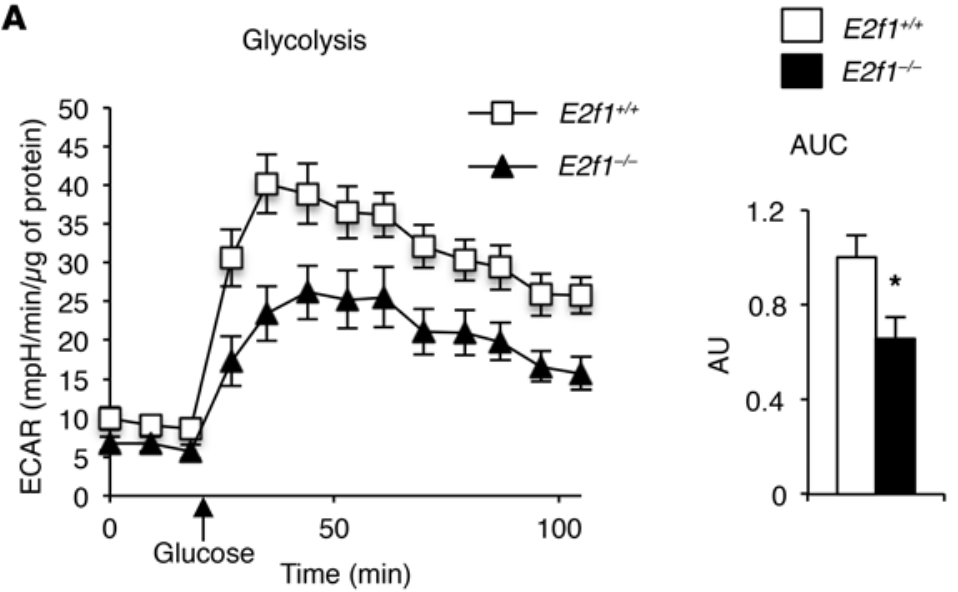

B

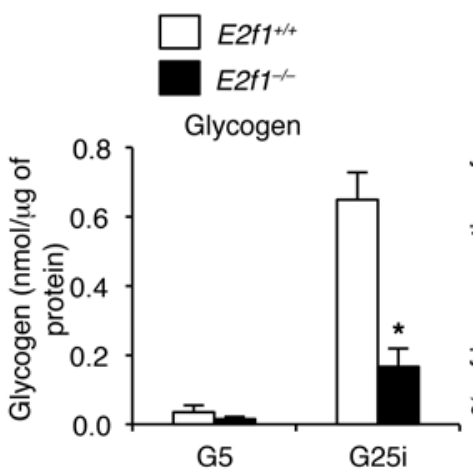

C

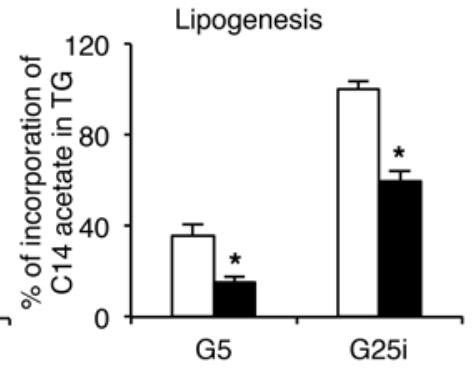

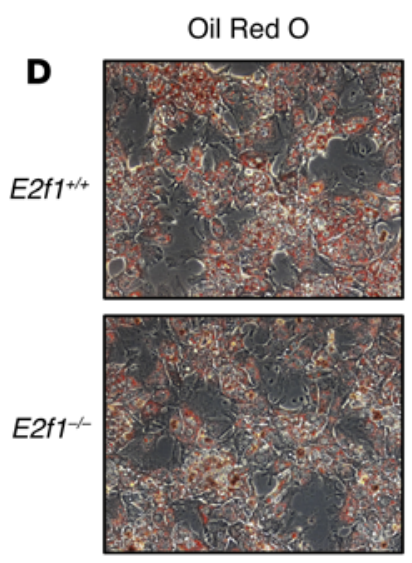

Figure 4. Glucose and lipid metabolism is impaired in E2f1/-/- hepatocytes. (A) ECAR of $E 2 \mathrm{f1}^{+/+}$and $\mathrm{E2} \mathrm{f1^{-/- }}$ hepatocytes after glucose treatment using a Seahorse analyzer ( 2 independent experiments, each with 8 technical replicates). (B) Glycogen content in $E 2 \mathrm{fr}^{+/+}$and $\mathrm{E} 2 \mathrm{f1} 1^{-/-}$hepatocytes treated for 24 hours with $G 5$ or C25i. (c) Quantification of the incorporation of C14-labeled acetate in the TC fraction in hepatocytes treated for 24 hours with $G 5$ or $\mathrm{C} 25 \mathrm{i}$ from the indicated genotypes, as a measure of lipogenesis. (D) Representative Oil Red $O$ staining of $\mathrm{E} \mathrm{f1}^{1^{/+}}$versus $\mathrm{E} 2 \mathrm{f1}^{-/-}$hepatocytes (original magnification, $\times 200$ ). Unless otherwise specified, all experiments represent the average of 3 independent experiments. ${ }^{*} P<0.05$ compared with control, by 2 -tailed, unpaired $t$ test. results suggested that CDK4, likely through E2F1 activation, regulates the expression of lipogenic genes.

E2F1 expression is increased in obese mice and humans. Increased glycolysis and de novo lipogenesis are typically observed during hepatic steatosis and hypertriglyceridemia development. Our results indicate that E2F1 regulates liver glucose and lipid metabolism, and we were therefore interested in elucidating the putative participation of E2F1 in liver pathology. Fatty liver is the first step in nonalcoholic fatty liver disease (NAFLD) and represents a risk factor for nonalcoholic steatohepatitis (NASH), fibrosis, cirrhosis, and hepatocellular carcinoma (20). Interestingly, in mice fed a high-fat diet and in $d b / d b$ mice, which constitute two models of hepatic steatosis, the mRNA expression levels of E2f1 were markedly increased compared with those in control animals (Figure 7, A and B). In addition, hepatic RB1 protein was hyperphosphorylated at Ser780 (Figure 7C). These results are consistent with increased E2F1 activity in these mouse models of fatty liver disease. In order to translate the findings obtained in mouse models to human pathology, we quantified the levels of E2F1 gene expression in liver biopsies from 10 lean patients versus levels in 10 obese, glucose-intolerant patients. The metabolic parameters for these patients are presented in Supplemental Table 1. A significant increase in E2F1 mRNA levels was observed in the liver of obese, glucose-intolerant patients compared with levels detected in the lean subjects, supporting our findings in mice (Figure 7D).

E2F1 deficiency protects against hepatic steatosis. To further demonstrate the participation of E2F1 in liver steatosis, we gen- erated $d b / d b E 2 f 1^{-/-}$mice. Deletion of $E 2 f 1$ in the $d b / d b$ model led to lower plasma insulin levels and BW (Figure 8A and Supplemental Figure 11A). No major differences in plasma glucose, FFA, or adiposity (fat mass content) were observed between $d b / d b E 2 \mathrm{fl}^{+/+}$ and $d b / d b E 2 f 1^{-/-}$mice, suggesting that $E 2 f 1$ deletion on the $d b / d b$ genetic background does not protect against diabetes or obesity, despite the misleading reduction in BW of these mice (Supplemental Figure 11, B and C). Indeed, $d b / d b E 2 f 1^{-/-}$mice have the classical features of obese $d b / d b$ mice, as they are hyperphagic, polydipsic, move less, and their respiratory exchange ratio (RER) is also reduced compared with $d b /+E 2 f 1^{+/+}$mice (Supplemental Figure 11, $\mathrm{D}$ and $\mathrm{E}$ ). However, we observed no differences in insulin sensitivity, as measured by an insulin tolerance test (ITT) (Supplemental Figure 11F), nor in the levels of AKT phosphorylation under basal conditions (Figure 8B) or after insulin stimulation (Supplemental Figure $11 G)$. In contrast, E2f1 deletion on the $d b / d b$ background resulted in decreased hepatic expression of lipogenic and glycolytic genes as well as decreased expression of SREBP1c and CHREBP at both the mRNA (Figure 8C) and protein (Figure 8D) levels compared with $d b / d b E 2 f 1^{+/+}$mice. This suggested that E2F1 participates in the regulation of lipogenic genes in the development of NAFLD in the $d b / d b$ model. Most important was the finding that $E 2 f 1$ deletion reversed the fatty liver phenotype in $d b / d b$ mice. Indeed, the livers of $d b / d b E 2 f 1^{-/-}$mice showed a normal macroscopic appearance compared with the pale and increased size of the livers of $d b / d b E 2 \mathrm{fl}^{+/+}$mice (Figure 9A). Moreover, $d b / d b E 2 f 1^{-/-}$livers had decreased weight compared with $d b / d b E 2 f 1^{+/+}$livers (Figure 9B) 
A

ChIP Ad-E2F1

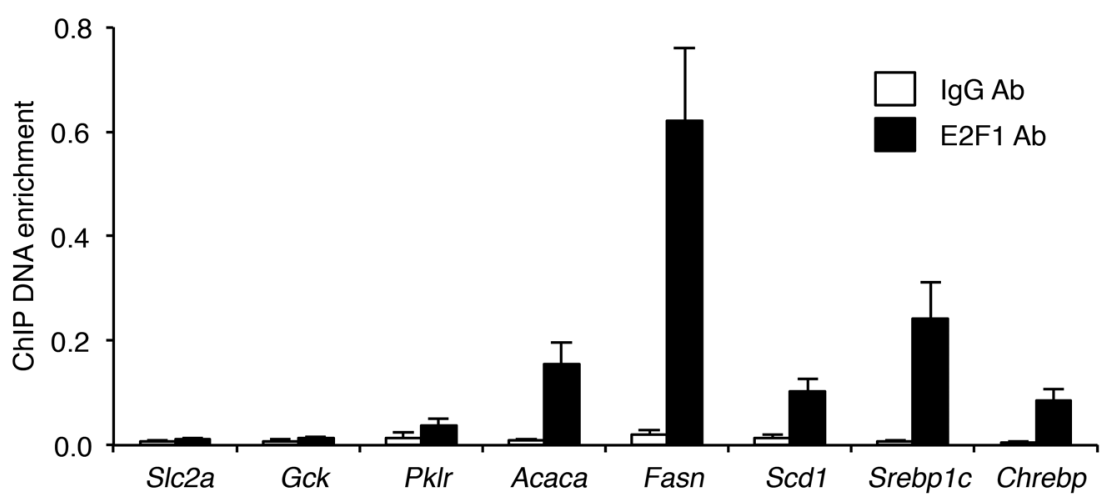

B

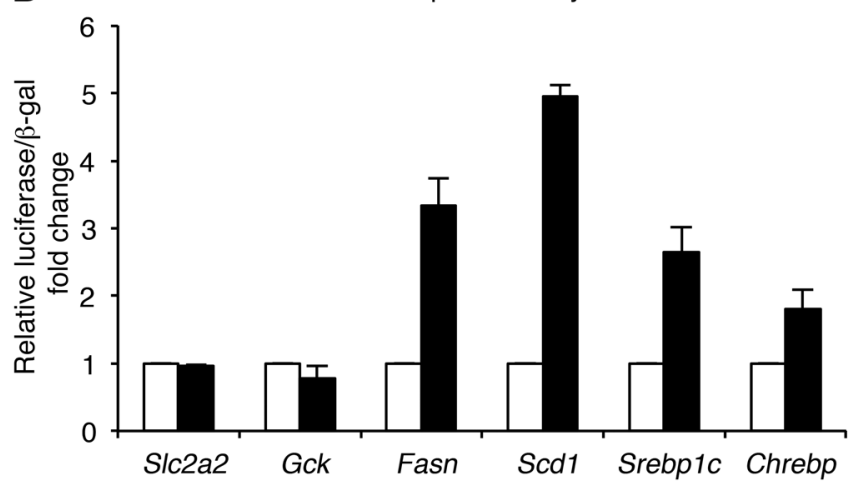

C

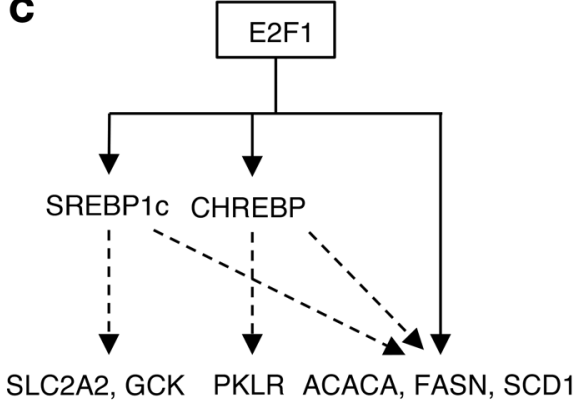

D
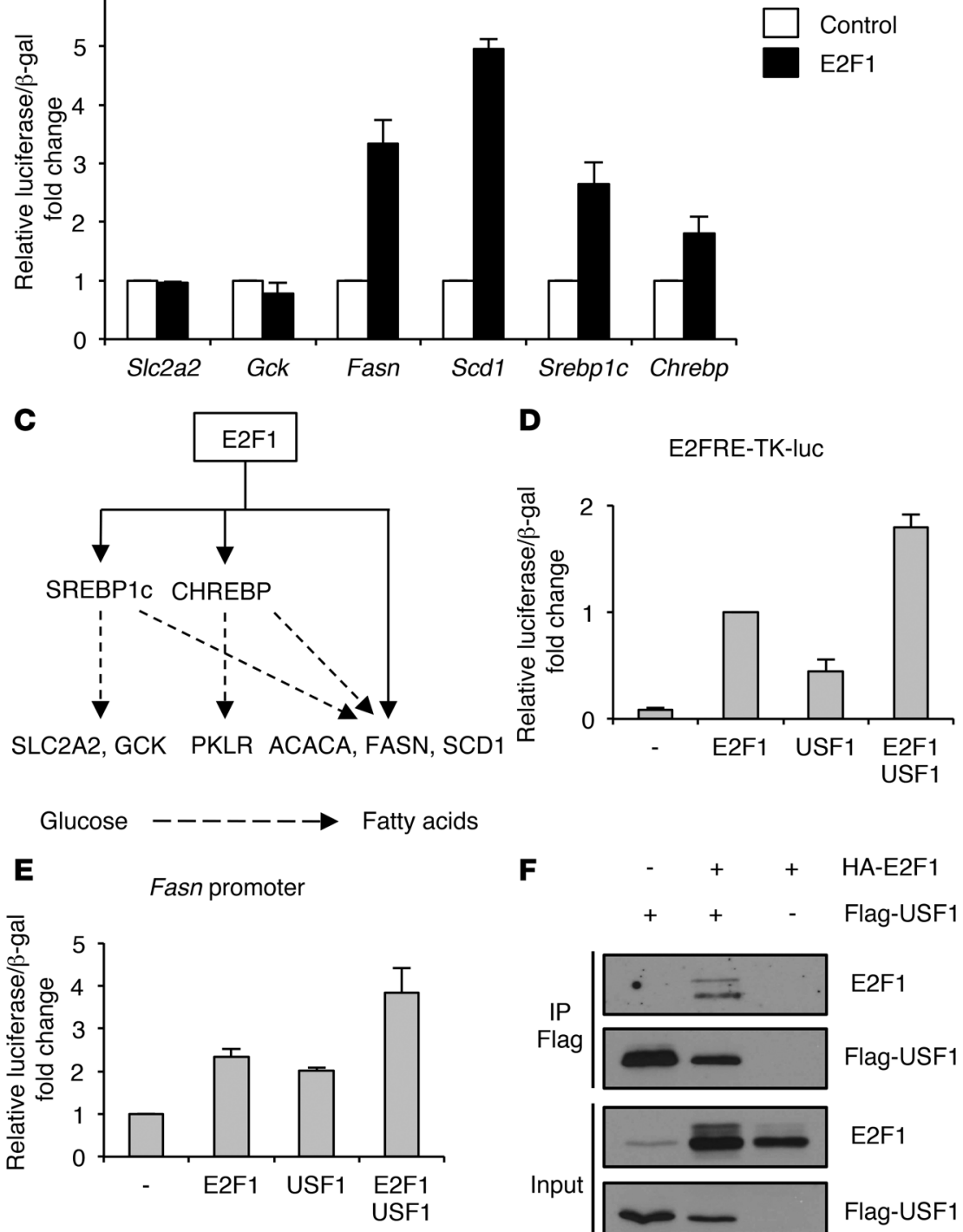

E2F1
$\mathbf{F}$
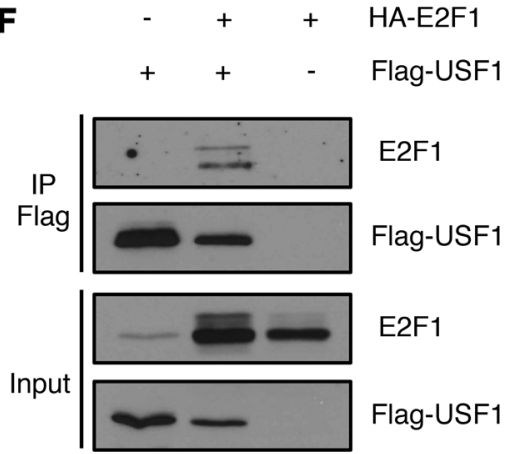

Figure 5. Lipogenic genes are bona fide E2F1 targets. (A) E2F1 ChIP on hepatocytes expressing Ad-E2F1 demonstrated E2F1 binding to the Acaca, Fasn, Scd1, Srebp1c, and Chrebp promoters, but not the SIc2a2 or Gck promoter $(n=5)$. (B) Luciferase-based reporter activity of the indicated promoters in HepG2 cells transfected with empty vector or the E2F1-HA expression vector. (C) Representative role of E2F1 in the transcriptional control of glycolysis and lipogenesis. E2F1 directly controlled Srebp1c, Chrebp, Acaca, Fasn, and Scd1 gene expression and indirectly controlled Slc2a2, Gck, and Pklr via SREBP1c and CHREBP. SREBP1c and CHREBP also participated in the control of Acaca, Fasn, and Scd1 gene expression. (D) E2F-TK-luc reporter activity in HepC2 hepatocytes transfected with empty vector, E2F1, or USF1. (E) Mouse Fasn promoter activity in hepatocytes transfected with empty vector, E2F1 or USF1. (F) Co-IP experiment on E2F1 and USF1 in HepG2 hepatocytes. Cells were transfected with E2F1-HA and Flag-USF1 as indicated, and protein was immunoprecipitated with a Flag Ab.

observed when lipogenesis is reduced. Finally, microscopic analysis indicated that E2F1 deficiency prevented hepatic steatosis (NAFLD) and the accumulation of intravesicular lipid droplets, which was observed with Oil Red O staining in hepatic steatosis (Figure 9F). These results suggest that $\mathrm{E} 2 \mathrm{~F} 1$ is an important factor facilitating the development of NAFLD.

Finally, to demonstrate that the observed effects were not the result of decreased insulin levels on the E2f1- background, we decided to also characterize obese $d b / d b E 2 \mathrm{fl}^{+/}$mice. These mice were similar to $d b / d b E 2 f^{+/+}$mice in terms of BW, plasma insulin levels, hyperphagia, water consumption, ambulatory movement, and RER (Supplemental Figure 12, A-D), whereas they were more glucose tolerant and insulin sensitive compared with $d b / d b E 2 f^{+/+}$mice (Supplemental Figure 12, E and F). This suggested that the effects of E2F1 are not secondary to decreased insulin levels or decreased weight. Furthermore, $d b / d b E 2 f 1^{+/}$mice had decreased hepatic steatosis, as illustrated by a lower liver TG content compared with that detected in $d b / d b \quad E 2 \mathrm{fl}^{+/+}$mice (Supplemental Figure 13B), but to a lesser extent than in $d b / d b E 2 \mathrm{fl}^{-/-}$mice (Figure 9C). These results were fully consistent with a reduction of the majority of glycolytic and lipogenic gene expression levels in the livers of $d b / d b E 2 f 1^{+/-}$ mice (Supplemental Figure 13A). and consistently showed a decrease in TG content (Figure 9C). Liver lipidomic analysis further demonstrated a strong decrease in palmitate and oleate fatty acids (Figure 9D). Consequently, the desaturation index of $d b / d b E 2 f^{-/-}$livers was also decreased compared with that of $d b / d b E 2 f 1^{+/+}$livers (Figure 9E), which is typically

\section{Discussion}

We show here that E2F1 is participates in hepatic glycolysis and de novo lipid synthesis through global transcriptional regulation of these pathways. Furthermore, we identify what we believe to be a 
A

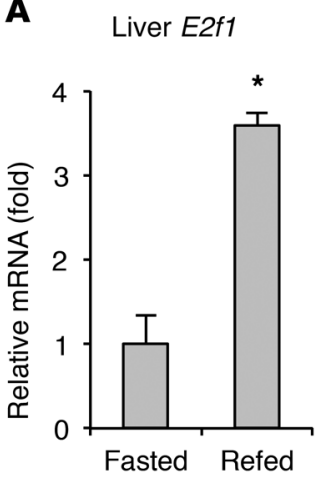

D

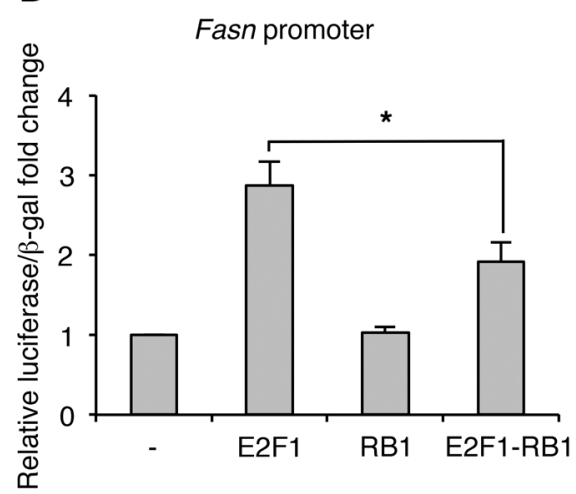

H $\square$ Ad sh-control Ad sh-CDK4

Cdk4

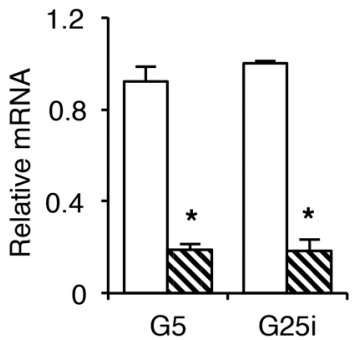

B
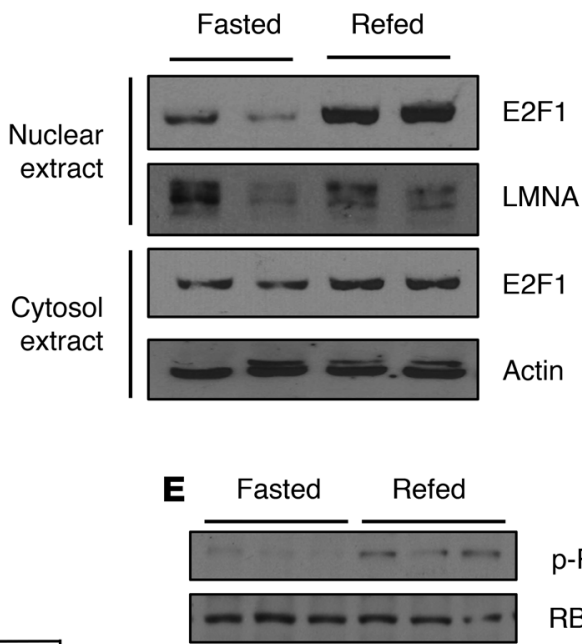

$\mathbf{F}$

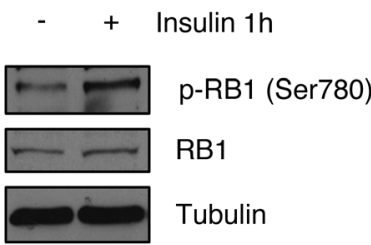
$R B 1$
C
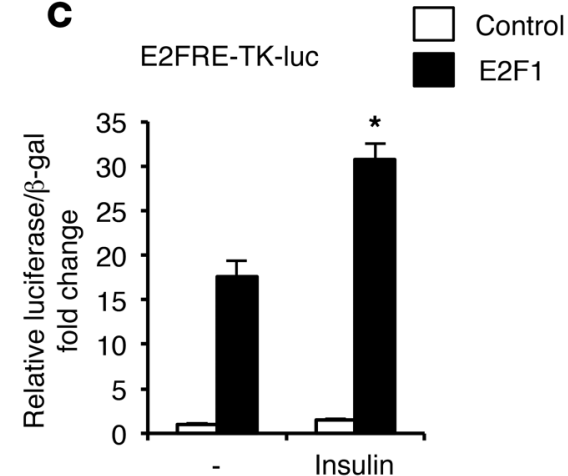

G p-RB1 (Ser780)

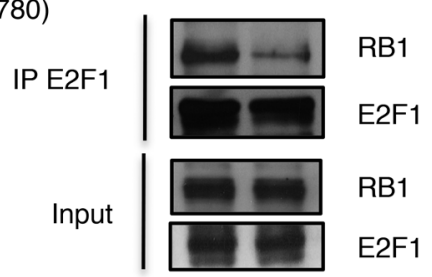

I sh-control $\frac{\text { sh-CDK4 }}{\text { G5 G25i }}$

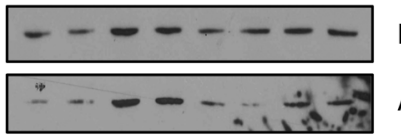

FASN ACACA

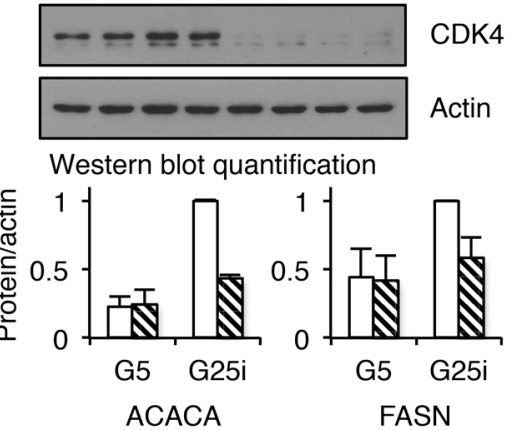

Figure 6. Insulin regulates E2F1 activity through RB1 and CDK4. (A) Relative E2f1 mRNA expression levels in the livers of mice after a 24-hour fast, followed by an 18-hour refeeding. (B) Protein expression analyses of the indicated cellular fractions in the livers of mice under the same conditions as in $\mathbf{A}$. (C) E2F reporter activity (E2FRE-TK-luc) in response to insulin in HEK293T cells. (D) Mouse Fasn promoter activity in HepG2 cells transfected with empty vector, E2F1, or RB1. (E) Ser780 phosphorylation of RB1 in the livers of fasted and refed mice. (F) Ser780 phosphorylation of RB1 in hepatocytes after 1 hour of insulin stimulation. (C) RB1 co-IP with E2F1 after 1 hour of insulin stimulation in HepG2 hepatocytes. (H) Relative mRNA expression of the indicated genes in hepatocytes treated with adenovirus expressing sh-control or sh-CDK4 and stimulated for 24 hours with G5 or C25i. (I) Protein levels of ACACA, FASN, and CDK4 in hepatocytes treated with adenovirus expressing sh-control or sh-CDK4 and stimulated for 24 hours with G5 or G25i. The experiments were performed at least 3 times. ${ }^{*} P<0.05$, by 2 -tailed, unpaired $t$ test.

new class of E2F1 targets that include ACACA, FASN, SCD1, SRE$\mathrm{BP} 1 \mathrm{c}$, and CHREBP, which are not directly involved in control of the cell cycle, proliferation, or apoptosis but rather are major regulators of metabolic pathways. Other genes such as those encoding the SLC2A2 transporter and GCK and PKLR glycolytic enzymes are also indirectly regulated by E2F1 through the SREBP1c and CHREBP transcription factors. These two transcription factors also participate in the E2F1-mediated control of Acaca, Fasn, and
$S c d 1$ gene expression. Interestingly, E2F1 expression and activity were increased in response to feeding and insulin levels in mouse livers and hepatocytes, respectively (Figure 6 and Supplemental Figure 9, A and B). This qualifies E2F1 as a major regulator of liver glucose and lipid metabolism (Figure $5 \mathrm{C}$ ). To our knowledge, only one additional transcription factor, $\operatorname{LXR} \alpha$, has been shown to regulate the same transcriptional program in the liver (15). This novel function of E2F1 was initially observed using a total KO. By using 
A

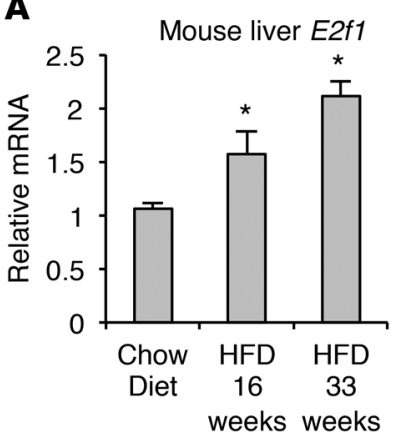

C

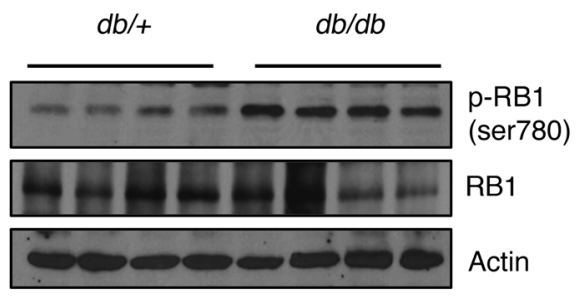

B

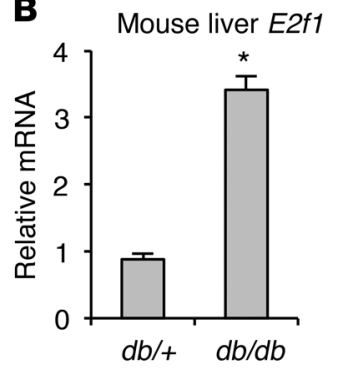

D

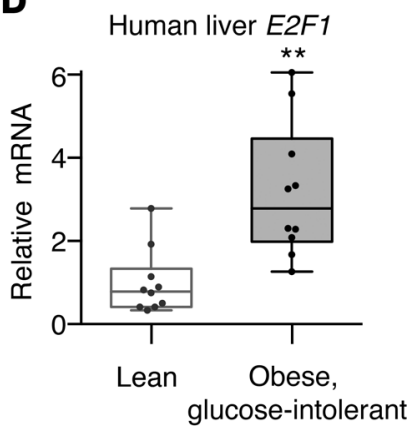

Figure 7. E2F1 gene expression is increased in obese mice and humans. (A) Relative expression of E2f1 mRNA in the livers of mice after 16 or 33 weeks on a high-fat diet. (B) E2f1 mRNA expression in the livers of control $d b /+$ and $d b / d b$ mice. (C) Ser780 phosphorylation of RB1 in the livers of $d b / d b$ mice. (D) Relative expression of E2F1 mRNA in livers of lean subjects and obese, glucose-intolerant patients. ${ }^{*} P<0.05$ and ${ }^{*} P<0.002$ compared with control, by 2 -tailed, unpaired $t$ test.

this model, we could not exclude the possibility that some of the effects observed in the absence of E2F1 were secondary, at least in part, to the role of E2F1 in insulin secretion (2). However, we demonstrated that this function was liver cell autonomous by using primary hepatocytes and E2F1 LKO mice fed a high-fructose diet. Even with residual liver E2F1 expression (Figure 2A), these mice clearly showed a decrease in glycolytic and lipogenic gene expression, leading to a reduction of hepatic steatosis.

De novo lipogenesis is a key regulatory pathway in liver and adipose tissue that facilitates the storage of excess energy in the form of lipids, typically as a result of increased glucose uptake. Fatty acid synthesis contributes, however poorly, to lipid content in the liver under normal physiological conditions (21), which is consistent with our observation that the effects of E2F1 on lipid synthesis in normal physiology are mild. Excess fat accumulation in the liver, such as that typically observed in obese, type 2 diabetic patients, can originate from increased fatty acid uptake, intensified fatty acid synthesis, reduced fatty acid oxidation, or decreased lipid export and transport. Nearly $30 \%$ of the TG content in the livers of NAFLD patients originates from de novo lipid synthesis (21). Interestingly, we found that E2F1 expression was significantly increased in the livers of obese, glucose-intolerant human subjects compared with that observed in normal-weight subjects (Figure 7D). Similar results were obtained in mouse models of fatty liver disease, in which E2F1 expression and activity were greatly increased, contributing to the augmentation of lipid content in the liver. Accordingly, our results showed that $E 2 f 1$ dele- tion in the $d b / d b$ mouse model of NAFLD, or in mice fed a high-sucrose diet, protected against lipid accumulation in the liver. However, decreased hepatic steatosis did not prevent marked hyperglycemia in $d b / d b$ E2 $\mathrm{fl}^{-/-}$mice, which is in contrast to the current knowledge that a reduction in hepatic TG levels correlates with improved insulin sensitivity $(22,23)$.

SREBP1c and CHREBP are essential activators of lipogenesis (12). In the context of NAFLD, it was reported that liver-specific KO of the gene encoding SCAP, which is necessary to promote SREBP1c nuclear activity, eliminates fatty liver in ob/ob diabetic mice (24). The same observations were reported for CHREBP downregulation in ob/ob mice using an adenoviral strategy (22). Since we show that E2F1 regulates the expression of SREBP1c and CHREBP, the abrogation of fatty liver in $d b / d b E 2 f^{-/}$mice could be at least partly due to the decreased expression of these transcription factors in the absence of E2F1.

Similar to our results, other studies have shown that liver steatosis can be regulated independently of insulin resistance. Liver-specific Irs1 ${ }^{-/}$mice, when fed a high-fat diet, were insulin resistant despite being protected from liver steatosis (25). Most interestingly, these mice showed near-normal liver histology and exhibited decreased nodular lesions compared with control mice and were protected from liver tumorigenesis (26). Other examples include the liver-specific phosphoinositide 3-kinase (PI3K) p110a-KO mice, which, similar to our findings in $E 2 \mathrm{fl}^{-/}$mice, were protected from hepatic steatosis without augmented plasma glucose and insulin levels when fed a high-fat diet (27). Conversely, insulin sensitization does not require amelioration of liver steatosis, as shown in CHREBPoverexpressing mice fed a high-fat diet, which were protected against insulin resistance despite increased lipid accumulation in the liver (28). Liver-specific Pten-KO mice also showed improved insulin sensitivity despite having liver steatosis (29). Indeed, insulin resistance is associated with increased lipid synthesis but also with increased glucose production in the liver. This indicates that lipogenesis could remain sensitive to insulin in the liver, whereas gluconeogenesis becomes resistant to inhibition by insulin according to a selective insulin resistance pathway, as suggested by Brown and Goldstein (30). It was demonstrated, however, that despite hyperglycemia and hyperinsulinemia, liver insulin receptor-KO (LIRKO) mice exhibit low plasma TG levels and no elevation of hepatic TG, suggesting that the divergent signaling pathway that controls lipid and glucose synthesis is downstream of the insulin receptor (31). Other studies in human subjects have also provided evidence to suggest that defects in insulin action in diabetic patients occur at the post-receptor level (32).

The paradox of "selective" insulin resistance raises the question of where the insulin-signaling regulatory pathway diverges from the gluconeogenic and lipogenic pathways. Elevated gluconeogenesis is explained by the lack of insulin repression due to an insulin-resistant state. However, there is a missing link between insulin resistance and the high rate of lipogenesis. It has been proposed that ER stress and inflammation could be 
A Plasma insulin

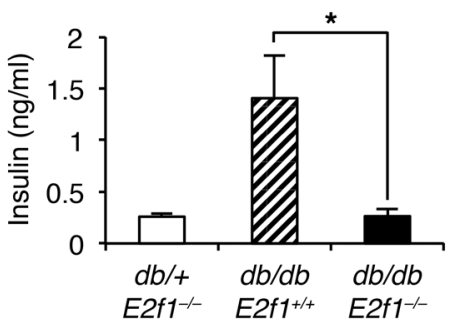

B

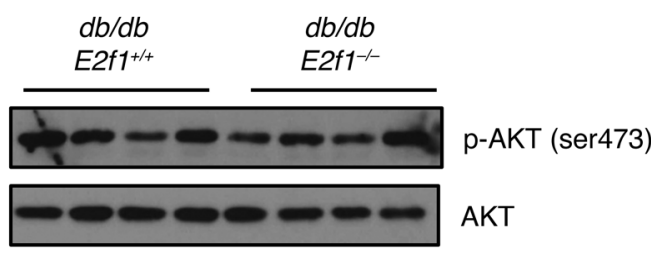

$d b /+E 2 f 1^{+/+}$

$\mathbb{Z} d b / d b$ E2f1+/+

$d b / d b E 2 f 1^{-\alpha}$

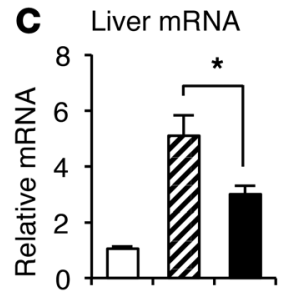

Slc2a2

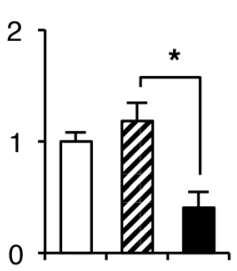

Gck

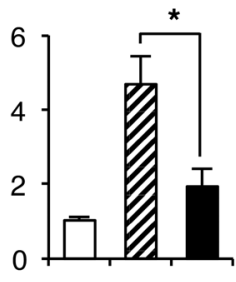

Acaca

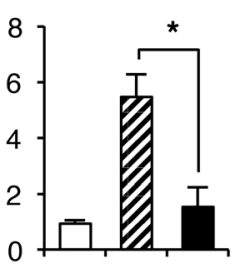

Fasn

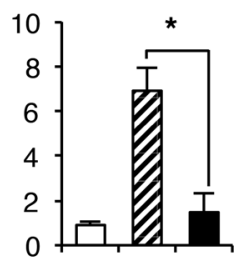

Scd1
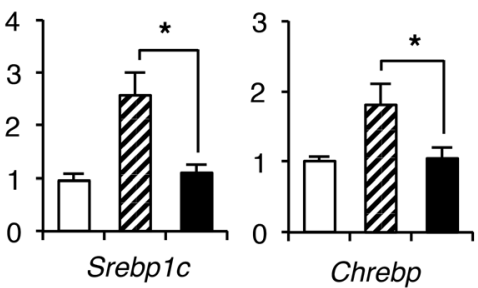

D
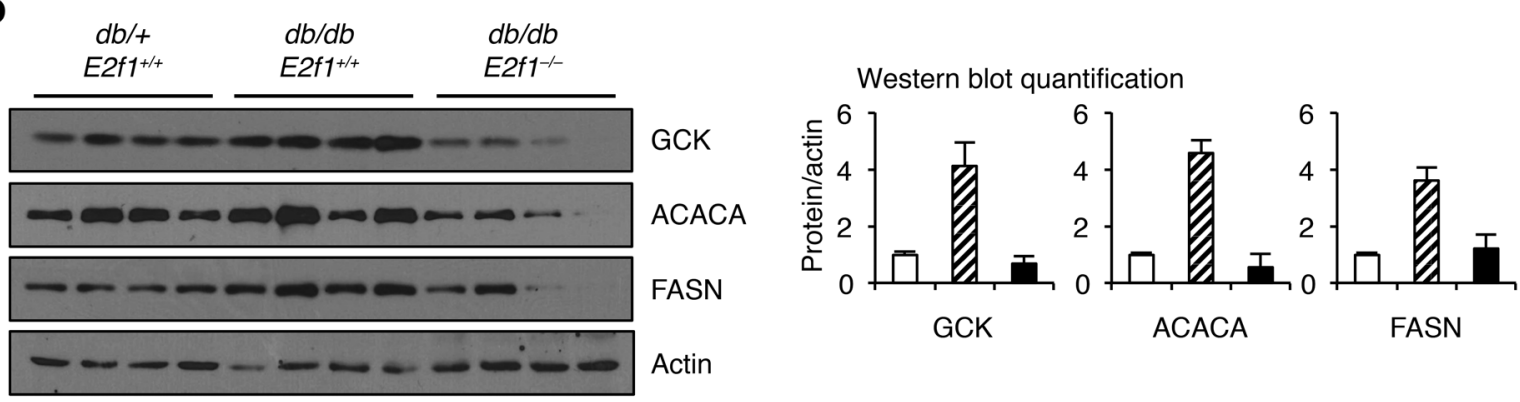

Figure 8. E2f1 deletion in $\mathbf{d b} / \mathbf{d b}$ mouse model decreases glycolytic and lipogenic programs. (A) Plasma insulin levels in $d b /+E 2 f 1^{+/+}, d b / d b E 2 f 1^{+/+}$, and $d b / d b$ E2f1-/- mice $(n=5-9)$. (B) Ser473 phosphorylation of AKT in the livers of $d b / d b E 2 f 1^{+/+}$and $d b / d b$ E2f1-/- mice. (C) Relative liver mRNA expression levels of the indicated genes in the annotated mouse genotypes $(n=4-6)$. (D) Western blot analyses of the expression of the indicated proteins in the livers of $d b /+E 2 f 1^{+/+}, d b / d b E 2 f 1^{+/+}$, and $d b / d b E 2 f 1^{-/-}$mice. ${ }^{*} P<0.05$, by 2-tailed, unpaired $t$ test.

involved in this abnormal regulation of liver function (33-35). ER stress has been reported to contribute to hepatic steatosis by supporting high lipogenic gene expression via SREBP1c and CHREBP activation (36). In our study, we demonstrate that liver E2F1 expression and activity were robustly increased in insulin-resistant $d b / d b$ mice and in obese, glucose-intolerant patients (Figure 7) and thus participates in liver steatosis. In this context, E2F1 could be activated by ER stress and, together with SREBP1c and CHREBP, maintain high lipogenesis.

E2F1 activity is increased in cancer cells, as this factor is essential for the transactivation of genes such as c-Myc and cyclin E, which regulate S-phase onset, DNA replication, and mitosis. Strikingly, de novo fatty acid biosynthesis is essential for dividing cells to synthesize new membranes. Lipogenesis also participates in the generation of signaling molecules, such as phosphatidylinositol, phosphatidylserine, and phosphatidylcholine, which are important in the activation of proliferative and survival pathways. Interestingly, it was shown that E2F1 participates in the control of lipid synthesis concomitantly with the regulation of proliferation in cancer cells (37). This was further demonstrated by the recent finding showing that fatty acid synthesis is coordinated with cell-cycle progression in proliferating cells (38). Moreover, a biphasic model of lipid accumulation, in which cycling cells show higher levels of lipids at the G1/S and G2/M transitions, was proposed $(10,38)$. The symmetrical effects of E2F1 on metabolic and proliferative pathways highlight the potential role of this cell-cycle regulator as a key mediator of the adapted metabolic response to proliferative stimuli, such as those observed during cancer cell transformation. Furthermore, our finding that E2F1 expression is increased in mouse models of diabetes and obesity could explain the increased cancer risk among obese and diabetic patients. Likewise, protection from E2F1-dependent hepatic steatosis may also protect against hepatic fibrosis and carcinogenesis $(10,11)$.

In summary, our results show that E2F1 mediates a metabolic switch that is notably increased to trigger lipid synthesis, most specifically under physiopathological conditions such as fatty liver disease.

\section{Methods}

Abs and biochemistry. The following Abs were obtained from Cell Signaling Technology: FASN (no. 3180); ACACA (no. 3662); lamin A/C (no. 2032); and phosphorylated RB (Ser780) (no. 8180). The following Abs were obtained from Santa Cruz Biotechnology Inc.: E2F1 (C-20; catalog sc-193); RB (C-15; catalog sc-50); RB (C-2; catalog sc-74562); CDK4 (C-22; catalog sc-260); and normal IgG rabbit (catalog sc-2027). The following Abs were obtained from Sigma-Aldrich: 
A

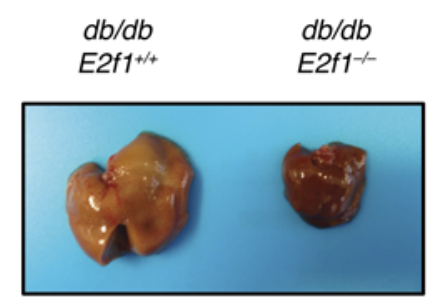

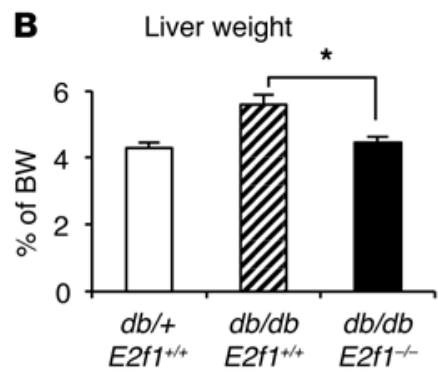

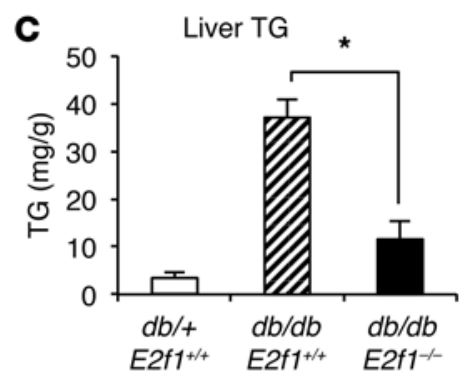

D

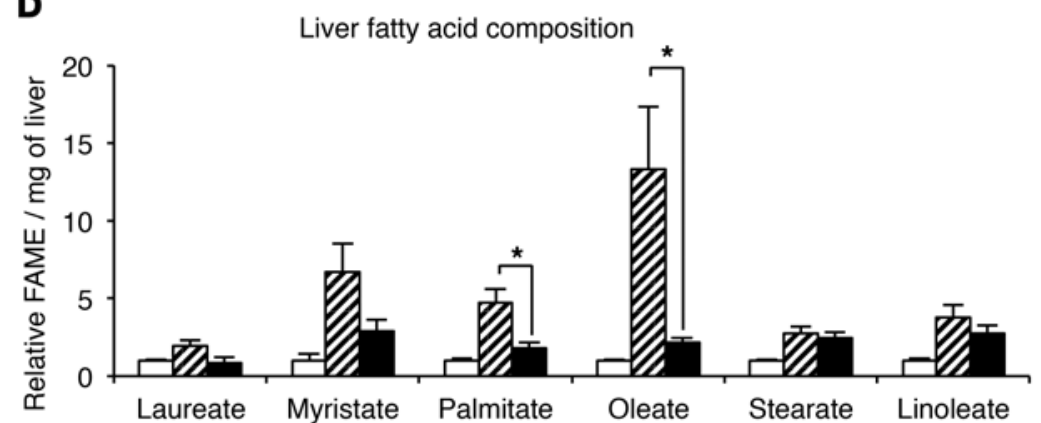

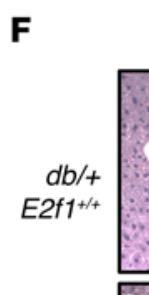

H\&E
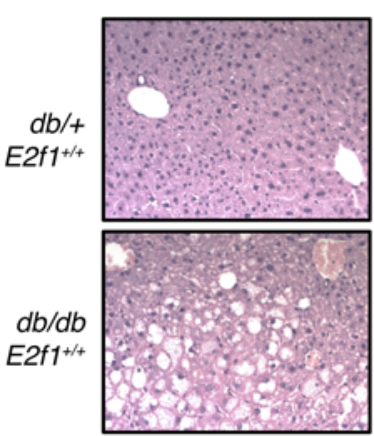

$d b /+E 2 f 1^{+/+}$
$d b / d b E 2 f 1^{+/+}$
$d b / d b E 2 f 1^{-1 /}$

\section{E Desaturation index}

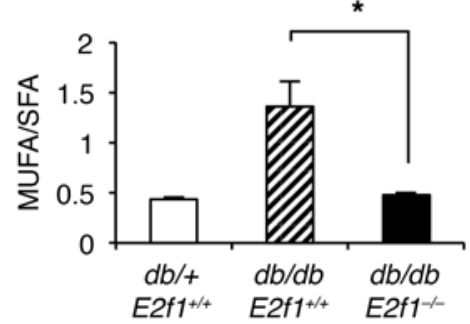

Oil

Red O
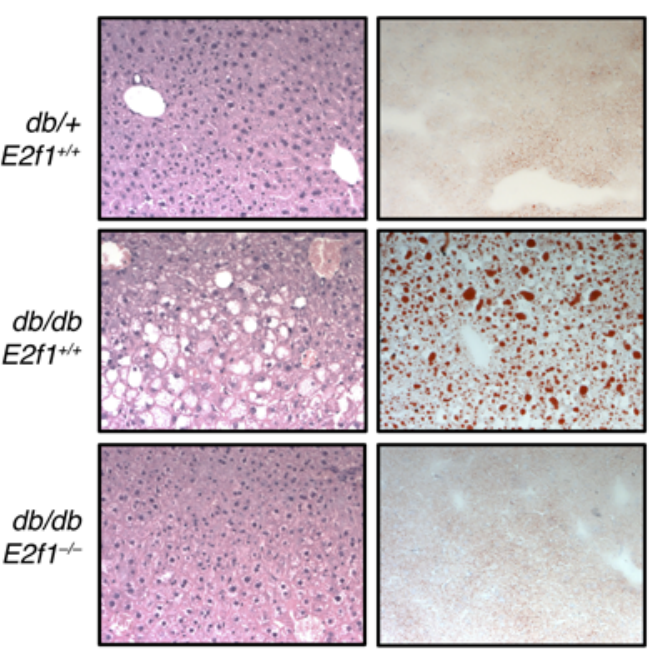

Figure 9. E2f1 deletion in the $d b / d b$ mouse model protects against hepatic steatosis. (A) Images of $d b / d b E 2 f 1^{-/-}$compared with $d b / d b E 2 F 1^{+/+}$mouse livers. (B) Liver weight expressed as a percentage of the total mass for the indicated genotypes $(n=5-9)$. (C) Quantification of liver TC in the indicated genotypes $(n=5-9)$. (D) Liver FAME analysis of $d b /+E 2 f 1^{+/+}, d b / d b E 2 f 1^{+/+}$, and $d b / d b E 2 f 1^{-/-}$mice $(n=4-7)$. (E) Desaturation index corresponded to the ratio of monounsaturated fatty acid:oleate C18:1/saturated fatty acid:palmitate C16 plus stearate C18 (MUFA/SFA) ratio. (F) Representative H\&E and Oil red 0 staining of liver sections from $d b /+E 2 f 1^{+/+}, d b / d b E 2 f 1^{+/+}$, and $d b / d b E 2 f 1^{-/-}$mice (original magnification, $\times 200$ ). ${ }^{*} P<0.05$, by 2-tailed, unpaired $t$ test.

$\beta$-actin (catalog A2066); tubulin (catalog T6199); and Flag-M2 (catalog F3165). The anti-GCK Ab was previously described (15). USF1 and USF2 Abs were a gift of B. Viollet (Institut Cochin, Paris, France) (39). Protein A agarose (Life Technologies) and anti-Flag M2 affinity gel (Sigma-Aldrich) were used for co-IP experiments. For subcellular fractionation, liquid N2 ground liver or cell pellets were washed in cold PBS and lysed using an NE-PER cell fractionation kit (Pierce, Thermo Scientific). Total protein extract and immunoblot analyses were performed as previously described (28).

DNA constructs and adenovirus. E2F1, RB, DP-1, E2FRE-TK-luc, and CMV- $\beta$-gal have been previously described (2). The mouse Gck and Slc2a2 promoters were a gift of K. Schoonjans (Ecole Polytechnique Fédérale de Lausanne [EPFL], Lausanne, Switzerland) and Y.H. Ahn (Yonsei University College of Medicine, Seoul, South Korea), respectively $(40,41)$. USF1 and USF2 plasmids were a gift of B. Viollet (Institut Cochin, Paris, France) (39). The human FASN promoter, human SCD1 promoter, human SREBP1c promoter, and human CHREBP promoter were obtained from SwitchGear Genomics. The mouse Fasn promoter was cloned in pGL3-basic by PCR from C57B6/J
DNA. The primer sequences were as follows: GAAGATCTTCCTGTCTGGGCTCTGGAGGCAGACGACAAGC (forward) and CCGAGCGTCGGAGGAATTTAAAGGGAGGGAGGAGAGGGT (reverse). E2F1 cDNA and CDK4 cDNA were inserted into the pAd-CMV-DEST-V5 adenoviral vector (Life Technologies). GFP adenovirus was a gift of J.R. Nevins (Duke University, Durham, North Carolina, USA), and sh-Cdk4 and sh-control adenoviruses were obtained from Vector BioLabs.

Cell culture and transfection. HEK293T and HepG2 cells were obtained from the American Type Culture Collection (ATCC) and respectively transfected using Lipofectamine 2000 reagent (Life Technologies) and X-tremeGENE (Roche). Primary hepatocytes were isolated as previously described (28). For experiments showing $E 2 \mathrm{fl}^{+/+}$ and $E 2 \mathrm{fl}^{-/-}$hepatocytes, cells were isolated from $E 2 \mathrm{fl}^{+/+}$and $E 2 \mathrm{fl}^{-/-}$ mice. For other experiment using hepatocytes, cells were isolated from C57Bl6 mice. C57Bl6 hepatocytes were not used as controls for $E 2 \mathrm{f1}^{-/-}$hepatocytes. Primary hepatocytes were obtained from C57Bl6J $\mathrm{E}_{21^{+/+}}$and $\mathrm{E} 2 \mathrm{fl}^{-/-}$mice. Mouse hepatocytes were harvested, cultured, and infected with adenoviruses as previously described (28). 
Hepatocyte glycolysis and lipogenesis. Glycolysis experiments were performed as previously described (40). Briefly, hepatocytes were seeded onto Seahorse XF24 plates (Seahorse Bioscience) and then glucose starved overnight. Cells were then washed and placed in an unbuffered DMEM-based medium containing $2 \mathrm{mM}$ glutamine for 1.5 hours. After ECAR, an indirect measurement of glycolysis was done with the Seahorse analyzer, and glucose $(25 \mathrm{mM})$ was injected into the cells directly with the Seahorse apparatus. For the lipogenesis experiment, hepatocytes were treated for 24 hours with low-glucose (G5) or high-glucose insulin (G25i). C14 acetate $(0.5 \mu \mathrm{Ci})$ was added for the last 2 hours. Incorporation of C14 into TG was measured by chromatography after lipid extraction and separation, following normalization for protein quantity.

ChIP and ChIP-seq experiments. Primary hepatocytes were infected with Ad-E2F1 after 36 hours, and cells were fixed for 10 minutes in $1 \%$ formaldehyde. Nuclear extracts were sonicated and precleared with protein A agarose/salmon sperm DNA. Chromatin was immunoprecipitated with anti-E2F1 c-20 Ab (catalog sc-193) or normal rabbit IgG (catalog sc-2027) (both from Santa Cruz Biotechnology Inc.). The full Western blot of E2F1 Ab is shown in Supplemental Figure 2, A and B. Immunoprecipitated chromatin was washed, reverse cross-linked, and purified with MinElute columns (QIAGEN). Quantification of E2F1 binding on a specific promoter was determined by qPCR (oligonucleotide sequences are listed in in Supplemental Table 1). Recoveries were calculated as a percentage of the input. For ChIP-seq, we performed two independent experimental replicates. Each replicate was the combination of two independent ChIP experiments. The Lausanne Genomic Technologies Facility (GTF) of UNIL generated the library and performed the sequencing (Illumina HiSeq 2500) of input and E2F1 ChIP (10 ng DNA). Sequencing alignment to the Ensembl Mouse Assembly NCBIM37 (mm9), peak detection, and peak assignment to genes were performed using the High-Throughput Sequencing portal HTSstation (17) developed at the Bioinformatics and Biostatistics Core Facility (BBCF) of the EPFL School of Life Sciences. The pipeline is available at http://htsstation.epfl.ch.

To assess the quality of the replicates and the reproducibility of the experiment, signals for both replicates were compared prior to combination (Supplemental Figure 2, C and D). Browser shots of input and E2F1 ChIP alignment are provided in Supplemental Figure 3. ChIP-seq peaks were determined using MACS software. A novel deconvolution algorithm was provided, which evaluates the shape of peaks within enriched regions found by MACS. This approach provides a more accurate estimation of binding-site locations and a lower number of false-positives (17). Using the DAVID website (http://david.abcc.ncifcrf.gov), we clustered E2F1 target genes with the general GOTERM_BP2 database. Data generated from ChIP-seq experiments are publicly available in the NCBI's Gene Expression Omnibus (GEO) database (GEO GSE74006).

Animal experiments and procedures. $E 2 \mathrm{fl}^{+/+}$and $\mathrm{E} 2 \mathrm{fl}^{-{ }^{--}}(\mathrm{B} 6 ; 129 \mathrm{~S} 4-$ E2f1tm1 Meg/J) mice were purchased from The Jackson Laboratory. C57BL/6J mice were obtained from Janvier Labs and were not used as controls for $E 2 \mathrm{fl}^{-/-}$mice. $E 2 \mathrm{fl}^{-/-}$mice and $\mathrm{db} /+$ mice (Janvier Labs) were crossed to obtain $E 2 f 1 d b$ mice. For the high-sucrose experiment, $30 \%$ sucrose (for 6 weeks), followed by $60 \%$ sucrose (for 3 weeks), was added to the drinking water, and the mice were euthanized after 4 hours of refeeding. E2f $f^{f l / f l}$ mice were generated by Tac-
onicArtemis (E2f1 ${ }^{\text {tm3110Arte }}$. The construct was designed to flank LoxP sites between $E 2 f 1$ exons 2 and 3 and to insert a positive selection marker (puromycin resistance) flanked by FRT sites in intron 1 as described in Supplemental Figure 14. After transfection into the TaconicArtemis C57BL/6N Tac ES cell line, homologous recombinant clones were isolated using positive (PuroR) and negative (TK) selection and expanded. After microinjection into BALB/c blastocysts, blastocysts were transferred into the uterine horn of 2.5-day postcoitum, pseudopregnant NMRI female mice. Highly chimeric mice were bred with females of the C57BL/6 strain. The E2fl-floxed allele was obtained after FLP-mediated removal of the selection marker. E2f1 liver-specific-KO (E2F1 LKO) mice were obtained after crossing E2f1-floxed mice with Alb-Cre mice, thus driving recombination in liver. Unless otherwise indicated in the figure legends, mice were sacrificed during the dark phase (fed condition).

Analytical procedures. TG serum concentrations were measured with a Hitachi robot (Roche) according to the manufacturer's instructions. Liver glycogen levels and TG content were determined as previously described (28)

Lipid analysis. Snap-frozen pieces of mouse liver were homogenized over dry ice in $50 \%$ aqueous methanol $\left(-20^{\circ} \mathrm{C}\right)$. Samples were snap-frozen and then thawed, and this was repeated 3 times. Samples were then centrifuged at $21,000 \mathrm{~g}$ for 10 minutes at $4^{\circ} \mathrm{C}$. Clarified supernatants were then transferred to fresh tubes on ice and were subjected to an addition of 2 volumes of amylene-stabilized chloroform at $-20^{\circ} \mathrm{C}$, vortexed, and then centrifuged at $21,000 \mathrm{~g}$ for 10 minutes at $4^{\circ} \mathrm{C}$. The chloroform phase was lyophilized and then used for fatty acid analysis. Fatty acid methyl esters (FAMEs) were generated by resuspending the lyophilized chloroform phase in $2 \% \mathrm{H}_{2} \mathrm{SO}_{4} /$ methanol and allowing transesterification to occur at $60^{\circ} \mathrm{C}$ for 4 hours. FAMEs were then isolated by adding 1 volume of hexane (ACS grade), vortexing, adding a one-tenth volume of saturated $\mathrm{NaCl}$, vortexing, and finally centrifuging the samples at room temperature at $21,000 \mathrm{~g}$ for 1 minute. The hexane phase was isolated and then lyophilized. Lyophilized FAMEs were resuspended in $50 \mu$ l hexane and subjected to gas chromatography-mass spectrometric (GC-MS) analysis. The GC temperature was maintained at $100^{\circ} \mathrm{C}$ for 5 minutes after the injection of $1 \mu \mathrm{l}$; the temperature was increased to $200^{\circ} \mathrm{C}$ by raising the temperature $15^{\circ} \mathrm{C}$ per minute, then the temperature was increased to $250^{\circ} \mathrm{C}$ by raising the temperature $5^{\circ} \mathrm{C}$ per minute, and finally, the temperature was increased to $300^{\circ} \mathrm{C}$ by raising the temperature $15^{\circ} \mathrm{C}$ per minute. The MS source and quadrupole tandem were held at $230^{\circ} \mathrm{C}$ and $150^{\circ} \mathrm{C}$. The detector ran in full-scan mode while recording ion abundances in the $100-650 \mathrm{~m} / z$ range.

Histology. Livers were fixed in $4 \%$ neutral buffered formalin and embedded in paraffin. Then, liver sections were cut and stained with H\&E. For the detection of neutral lipids, liver cryosections were stained using the Oil Red $\mathrm{O}$ technique.

mRNA analysis. mRNAs from liver and primary hepatocytes were isolated using an RNeasy Kit (QIAGEN) and then reverse transcribed. qPCR analysis was performed using a 7900HT Fast Real-Time PCR System (Applied Biosystems) and SYBR Green detection of the amplified products. The relative quantification for a given gene was corrected to the cyclophilin B and RS9 mRNA values (oligonucleotide sequences are provided in Supplemental Table 1).

Humans samples. All obese patients included in this study were from the Atlas Biologique de l'Obésité Sévère (ABOS) study. This 
study also includes a group of lean and normoglycemic control patients who had surgery for benign and noninflammatory pathologies. Clinical data were collected at the Centre Hospitalier Régional Universitaire de Lille. The obese patients were morbidly obese (BMI $>40$ ) and glucose intolerant. The metabolic parameters for the two groups are presented in Supplemental Table 1. Liver samples were collected during surgery within the first 15 minutes of the procedure, weighed, and snap-frozen in liquid nitrogen. Total RNA and protein were extracted from liver samples $(10 \mathrm{mg})$ using the Allin-One Purification Kit (Norgen Biotek Corp.). First-strand cDNA synthesis was performed using $500 \mathrm{ng}$ total RNA as a template and Superscript II reverse transcriptase (Life Technologies) primed with 50 pmol random hexamers (New England BioLabs). qPCR was performed using the Bio-Rad MyiQ Single-Color Real-Time PCR Detection System and Bio-Rad iQ SYBR Green Supermix (Bio$\mathrm{Rad})$. Gene expression was normalized to two housekeeping genes ( $A C T B$, encoding eta-actin, or RPS9, encoding ribosomal protein S9). Relative mRNA fold changes between groups were calculated using the $\Delta$ Ct method.

Statistics. Results are expressed as the mean \pm SEM. Statistical significance was assessed with a 2-tailed, unpaired $t$ test using Prism 6 software (GraphPad Software). Differences were considered statistically significant at $P<0.05$. All experiments were performed on at least three independent occasions unless otherwise stated.

Study approval. All animal care and treatment procedures were performed in accordance with Swiss guidelines and were approved by the Canton of Vaud, Service de la Consommation et des Affaires Vétérinaires (SCAV) (authorization VD 2627.b). The protocol concerning the use of biopsies from human subjects was performed in agreement with
French regulations and approved by the institutional ethics committees of the University of Lille and the Centre Hospitalier Régional Universitaire de Lille (Clinicaltrials.gov NCT01129297). All patients provided written informed consent to participate in this study.

\section{Author contributions}

PDD and LF conceptualized the study and designed the experiments. PDD, ICLM, AGC, QL, EB, BD, and JSA performed the experiments. BNN and NJD performed lipidomics analysis. CB and FP performed the human study.

\section{Acknowledgments}

We acknowledge the members of the Fajas laboratory for their support and discussions. We thank A. Chanthany and A.C. Thomas for their technical support. We also thank Jacques Rougemont, Marion Leleu, and Sara Benmohammed of the BBCF at the EPFL for their assistance with ChIP-seq analysis. This work was supported by grants from the Swiss Ligue Contre le Cancer, the French Ligue Contre le Cancer, the Swiss National Science Foundation, and the Fondation de France.

Address correspondence to: Lluis Fajas, Department of Physiologie, Université de Lausanne. CH-1005 Lausanne, Switzerland. Phone: 41.21.692.55.10; E-mail: 1luis.fajas@unil.ch.

Jean-Sébastien Annicotte's present address is: European Genomic Institute for Diabetes, Université Lille Nord de France, UMR 8199 CNRS, Lille, France.
1. Dimova DK, Dyson NJ. The E2F transcriptional network: old acquaintances with new faces. Oncogene. 2005;24(17):2810-2826.

2. Annicotte JS, et al. The CDK4-pRB-E2F1 pathway controls insulin secretion. Nat Cell Biol. 2009;11(8):1017-1023.

3. Fajas L, Landsberg RL, Huss-Garcia Y, Sardet C, Lees JA, Auwerx J. E2Fs regulate adipocyte differentiation. Dev Cell. 2002;3(1):39-49.

4. Blanchet E, et al. E2F transcription factor-1 regulates oxidative metabolism. Nat Cell Biol. 2011;13(9):1146-1152.

5. Hsieh MC, Das D, Sambandam N, Zhang $\mathrm{MQ}$, Nahle Z. Regulation of the PDK4 isozyme by the Rb-E2F1 complex. J Biol Chem. 2008;283(41):27410-27417.

6. Fernandez de Mattos S, Lam EW, Tauler A. An E2F-binding site mediates the activation of the proliferative isoform of 6-phosphofructo-2-kinase/fructose-2,6-bisphosphatase by phosphatidylinositol 3-kinase. Biochem $J$. 2002;368(pt 1):283-291.

7. Dali-Youcef N, et al. Adipose tissue-specific inactivation of the retinoblastoma protein protects against diabesity because of increased energy expenditure. Proc Natl Acad Sci U S A. 2007;104(25):10703-10708.

8. Nicolay BN, et al. Loss of RBF1 changes glutamine catabolism. Genes Dev. 2013;27(2):182-196.

9. Lopez-Mejia IC, Fajas L. Cell cycle regulation of mitochondrial function. Curr Opin Cell Biol.
2015;33:19-25.

10. Conner EA, Lemmer ER, Omori M, Wirth PJ, Factor VM, Thorgeirsson SS. Dual functions of E2F-1 in a transgenic mouse model of liver carcinogenesis. Oncogene. 2000;19(44):5054-5062.

11. Zhang Y, et al. E2F1 is a novel fibrogenic gene that regulates cholestatic liver fibrosis through the Egr-1/SHP/EID1 network. Hepatology. 2014;60(3):919-930.

12. Postic C, Girard J. Contribution of de novo fatty acid synthesis to hepatic steatosis and insulin resistance: lessons from genetically engineered mice. JClin Invest. 2008;118(3):829-838.

13. Brown MS, Goldstein JL. The SREBP pathway: regulation of cholesterol metabolism by proteolysis of a membrane-bound transcription factor. Cell. 1997;89(3):331-340.

14. Foretz M, Guichard C, Ferré P, Foufelle F. Sterol regulatory element binding protein $1 \mathrm{c}$ is a major mediator of insulin action on the hepatic expression of glucokinase and lipogenesis-related genes. Proc Natl Acad Sci U S A. 1999;96(22):12737-12742.

15. Denechaud PD, et al. ChREBP, but not LXRs, is required for the induction of glucoseregulated genes in mouse liver. JClin Invest. 2008;118(3):956-964.

16. Chan SM, et al. Activation of PPAR $\alpha$ ameliorates hepatic insulin resistance and steatosis in high fructose-fed mice despite increased endoplasmic reticulum stress. Diabetes. 2013;62(6):2095-2105
17. David FP, et al. HTSstation: a web application and open-access libraries for high-throughput sequencing data analysis. PLoS One. 2014;9(1):e85879.

18. Cao AR, Rabinovich R, Xu M, Xu X, Jin VX, Farnham PJ. Genome-wide analysis of transcription factor E2F1 mutant proteins reveals that $\mathrm{N}$ - and $\mathrm{C}$-terminal protein interaction domains do not participate in targeting E2F1 to the human genome. J Biol Chem. 2011;286(14):11985-11996.

19. Wong RH, Chang I, Hudak CS, Hyun S, Kwan HY, Sul HS. A role of DNA-PK for the metabolic gene regulation in response to insulin. Cell. 2009;136(6):1056-1072.

20. Yeh MM, Brunt EM. Pathological features of fatty liver disease. Gastroenterology. 2014;147(4):754-764.

21. Donnelly KL, Smith CI, Schwarzenberg SJ, Jessurun J, Boldt MD, Parks EJ. Sources of fatty acids stored in liver and secreted via lipoproteins in patients with nonalcoholic fatty liver disease. J Clin Invest. 2005;115(5):1343-1351.

22. Dentin R, et al. Liver-specific inhibition of ChREBP improves hepatic steatosis and insulin resistance in ob/ob mice. Diabetes. 2006;55(8):2159-2170.

23. Neschen S, et al. Prevention of hepatic steatosis and hepatic insulin resistance in mitochondrial acyl-CoA:glycerol-sn-3-phosphate acyltransferase 1 knockout mice. Cell Metab. 2005;2(1):55-65. 
24. Moon YA, et al. The Scap/SREBP pathway is essential for developing diabetic fatty liver and carbohydrate-induced hypertriglyceridemia in animals. Cell Metab. 2012;15(2):240-246.

25. Guo S, et al. The Irs1 branch of the insulin signaling cascade plays a dominant role in hepatic nutrient homeostasis. Mol Cell Biol. 2009;29(18):5070-5083.

26. Nakamura A, et al. Protection from non-alcoholic steatohepatitis and liver tumourigenesis in high fat-fed insulin receptor substrate-1-knockout mice despite insulin resistance. Diabetologia. 2012;55(12):3382-3391.

27. Chattopadhyay M, Selinger ES, Ballou LM, Lin RZ. Ablation of PI3K p110-alpha prevents high-fat diet-induced liver steatosis. Diabetes. 2011;60(5):1483-1492.

28. Benhamed F, et al. The lipogenic transcription factor ChREBP dissociates hepatic steatosis from insulin resistance in mice and humans. J Clin Invest. 2012;122(6):2176-2194.

29. Horie Y, et al. Hepatocyte-specific Pten deficiency results in steatohepatitis and hepatocellular carcinomas. J Clin Invest. 2004;113(12):1774-1783.

30. Brown MS, Goldstein JL. Selective versus total insulin resistance: a pathogenic paradox. Cell Metab. 2008;7(2):95-96.

31. Biddinger SB, et al. Hepatic insulin resistance is sufficient to produce dyslipidemia and susceptibility to atherosclerosis. Cell Metab. 2008;7(2):125-134.

32. Semple RK, et al. Postreceptor insulin resistance contributes to human dyslipidemia and hepatic steatosis. J Clin Invest. 2009;119(2):315-322.

33. Wellen KE, Hotamisligil GS. Inflammation, stress, and diabetes. JClin Invest. 2005;115(5):1111-1119.

34. Hotamisligil GS. Inflammation and metabolic disorders. Nature. 2006;444(7121):860-867.

35. Ozcan U, et al. Chemical chaperones reduce ER stress and restore glucose homeostasis in a mouse model of type 2 diabetes. Science. 2006;313(5790):1137-1140.

36. Kammoun HL, et al. GRP78 expression inhibits insulin and ER stress-induced SREBP-1c activa- tion and reduces hepatic steatosis in mice. J Clin Invest. 2009;119(5):1201-1215.

37. Bhatia B, Hsieh M, Kenney AM, Nahle Z. Mitogenic Sonic hedgehog signaling drives E2F1-dependent lipogenesis in progenitor cells and medulloblastoma. Oncogene. 2011;30(4):410-422.

38. Scaglia N, Tyekucheva S, Zadra G, Photopoulos C, Loda M. De novo fatty acid synthesis at the mitotic exit is required to complete cellular division. Cell Cycle. 2014;13(5):859-868.

39. Viollet B, Lefrancois-Martinez AM, Henrion A, Kahn A, Raymondjean M, Martinez A. Immunochemical characterization and transacting properties of upstream stimulatory factor isoforms. J Biol Chem. 1996;271(3):1405-1415.

40. Oosterveer MH, et al. LRH-1-dependent glucose sensing determines intermediary metabolism in liver. J Clin Invest. 2012;122(8):2817-2826.

41. Im SS, et al. Glucose-stimulated upregulation of GLUT2 gene is mediated by sterol response element-binding protein-1c in the hepatocytes. Diabetes. 2005;54(6):1684-1691. 